\title{
A Teoria de Duffin-Kemmer-Petiau
}

\author{
The Theory of Duffin-Kemmer-Petiau
}

\author{
T.R. Cardoso, B.M. Pimentel \\ Instituto de Física Teórica (IFT), UNESP, São Paulo State University, Rua Doutor Bento Teobaldo Ferraz 271, \\ Bloco II, Barra Funda CEP 01140-070, São Paulo, Brazil
}

Recebido em 30 de março de 2016. Aceito em 22 de maio de 2016

\begin{abstract}
A teoria de Duffin-Kemmer-Petiau é apresentada a partir de um contexto histórico, realçando as ideias e analogias para seu desenvolvimento e conduzindo de forma intuitiva a sua álgebra e propriedades gerais. A equivalência entre este formalismo e os formalismos de Klein-Gordon-Fock e Proca para os casos livre e minimamente acoplado, como também a problemática em torno do limite $m=0$ são expostas. Este estudo se completa com a riqueza nas possíveis interações fenomenológicas que a teoria de Duffin-Kemmer-Petiau permite, tornando toda a teoria uma fonte de inspiração e cultura.

Palavras-chave: teoria de Duffin-Kemmer-Petiau, álgebra de Duffin-Kemmer-Petiau, equação de KleinGordon-Fock, equações de Proca, equações de primeira ordem.
\end{abstract}

The theory of Duffin-Kemmer-Petiau is presented from a historical point of view, highlighting the ideas and analogies which led to its formal development, as well as to its algebra and general properties. Furthermore, some misunderstood concepts surrounding the massless limit, $m=0$, as well as the equivalence between this formalism and the formalisms of Klein-Gordon-Fock and Proca, for free and minimal coupling cases, are exposed. Insofar the completion of this study with the richness of the possible phenomenological interactions that make the theory of Duffin-Kemmer-Petiau a source of inspiration and culture.

Keywords: Duffin-Kemmer-Petiau theory, Duffin-Kemmer-Petiau algebra, Klein-Gordon-Fock equation, Proca equations, first-order equation.

\section{Inspirações para o desenvolvimento da teoria}

A história do átomo de hidrogênio está intimamente relacionada ao desenvolvimento da Física Quântica [1,2]. A estrutura bruta, assim chamadas as primeiras linhas espectrais do átomo de hidrogênio, foram interpretadas teoricamente por Bohr através de princípios clássicos e pressupostos quânticos ainda muito prematuros à época [3]. De fato, apenas com o advento da equação de Schrödinger [4 foi possível adquirir o espectro de energia do elétron em um potencial coulombiano gerado por um próton estático, ou o átomo de hidrogênio, plenamente fundamentado em princípios da Mecânica Quântica

$$
E_{n}^{(0)}=-\left[\frac{m}{2 \hbar^{2}} Z^{2} e^{4}\right] \frac{1}{n^{2}}
$$

que, em termos da constante de estrutura fina, $\alpha$,

$$
\alpha \equiv \frac{e^{2}}{\hbar c} \simeq \frac{1}{137}
$$

se reescreve como

$$
E_{n}^{(0)}=-\frac{1}{2 n^{2}} Z^{2} \alpha^{2} m c^{2}
$$

em que $n=1,2,3, \ldots$ Esta equação também é conhecida como o espectro de Bohr - que a obteve em 1913; de fato, a equação de Schrödinger é posterior a 1920. Altamente degenerado, este espectro já apresenta em seu primeiro estado excitado a superposição de três estados, ${ }^{2} S_{1 / 2},{ }^{2} P_{1 / 2}$ e ${ }^{2} P_{3 / 2}$, todos com energia proporcional à $\alpha^{2} m c^{2}$.

Com o aumento na precisão dos experimentos de aquisição do espectro, novas linhas espectrais foram sendo descobertas e estados antes degenerados

\footnotetext{
*Endereço de correspondência: tirdona@gmail.com.
} 
agora apresentavam tênues divisões, quebrando sua degenerescência [2] Na ausência de um fundamento teórico que possibilitasse uma interpretação coesa de todas as minúcias do espectro, foram incluídos "à mão"termos na hamiltoniana de Schrödinger que justificassem as discrepâncias do espectro, tais como a interação spin-órbita e correções relativísticas, ambas com energias da ordem de $\alpha^{4} m c^{2}$, cuja contribuição ficou conhecida como estrutura fina.

Ainda menor, em ordem $\left(\frac{m}{m_{p}}\right) \alpha^{4} m c^{2}$, são efeitos devido à interação magnética entre os momentos de dipolo do elétron e próton, o que ficou conhecido como estrutura hiperfina.

Contudo, em 1928 Dirac propõe sua teoria quântica para o elétron [5] em que a estrutura fina do átomo de hidrogênio aparece naturalmente, fruto do estudo do elétron sob a ação de um potencial coulombiano (próton). Em verdade, o limite nãorelativístico da equação de Dirac com este potencial leva à equação de Schrödinger acrescida do termo de spin-órbita. Assim, a teoria desenvolvida por Dirac pareceu então ter resolvido todas as particularidades do espectro do átomo de hidrogênio à sua época. $\mathrm{E}$ esse foi, sem dúvida, um dos grandes triunfos de sua teoria.

Qual seria a motivação de Dirac para encontrar o que ficou conhecido como a equação de Dirac? Resolver o célebre problema da densidade de probabilidade na teoria de Klein-Gordon-Fock.

A equação de Schrödinger pode ser obtida através da relação energia-momento não-relativística clássica $E=\frac{p^{2}}{2 m}$, na promoção da energia $E$ e o momento $\vec{p}$ a operadores, $i \hbar \frac{\partial}{\partial t}$ e $-i \hbar \vec{\nabla}$, respectivamente, atuando sobre uma função de onda complexa $\Psi(\vec{r}, t)$. Esta função de onda pode então ser associada a uma partícula, de tal forma que $|\Psi(\vec{r}, t)|^{2} d^{3} x$ é a densidade de probabilidade de encontrar esta partícula em um elemento de volume $d^{3} x$. Esta interpretação é possível, haja vista que a densidade $\rho$, $|\Psi(\vec{r}, t)|^{2}$ (que é positiva-definida), e a densidade de corrente $\vec{J}$ satisfazem uma equação de continuidade $\partial \rho / \partial t+\vec{\nabla} \cdot \vec{J}=0$. Assim, $\rho$ pode ser interpretado como uma densidade de probabilidade e $\vec{J}$ como uma corrente de probabilidade.

Uma construção análoga à equação de Schrödinger para a relação energia-momento relativística,
$E^{2}=p^{2} c^{2}+m^{2} c^{4}$, conduz à equação de KleinGordon-Fock 1$]$

$$
-\frac{1}{c^{2}} \frac{\partial^{2} \Phi(\vec{r}, t)}{\partial t^{2}}+\nabla^{2} \Phi(\vec{r}, t)-\frac{m^{2} c^{2}}{\hbar^{2}} \Phi(\vec{r}, t)=0
$$

ou ainda

$$
\left[\square+\frac{m^{2} c^{2}}{\hbar^{2}}\right] \Phi(\vec{r}, t)=0
$$

que, todavia, conduz a uma expressão para a densidade $\rho$ dada por

$$
\rho \equiv \frac{i \hbar}{2 m c^{2}}\left[\Phi^{*}(\vec{r}, t) \frac{\partial \Phi(\vec{r}, t)}{\partial t}-\Phi(\vec{r}, t) \frac{\partial \Phi^{*}(\vec{r}, t)}{\partial t}\right]
$$

que não é positiva-definida e, logo, não pode ser interpretada como uma densidade de probabilidad $\mathrm{L}^{2}$ Assim, existia uma busca por uma equação de primeira ordem na derivada temporal que conduzisse a uma densidade de probabilidade positiva-definida. Partindo desse pressuposto, Dirac então determina uma equação em primeira ordem

$$
\left(i \gamma^{\mu} \partial_{\mu}-m\right) \psi(x)=0
$$

cuja álgebra para as matrizes $\gamma$ é dada por

$$
\gamma^{\mu} \gamma^{\nu}+\gamma^{\nu} \gamma^{\mu}=2 g^{\mu \nu}
$$

com densidade de probabilidade positiva-definida

$$
\rho \equiv \psi^{\dagger} \psi \text {. }
$$

Diante do enorme sucesso da teoria de Dirac, de Broglie sugere que o fóton poderia ser formado pela combinação de dois léptons e que esta combinação seria então a responsável por atribuir massa ao fóton $[8]$. Com efeito, essa premissa era compartilhada por muitos cientistas à época. Tomado por esta ideia e com profundo conhecimento da estrutura algébrica da equação de Dirac, de Broglie inicia sua busca por uma equação de primeira ordem na esperança de obter uma equação para o seu fóton massivo. Petiau, um dos estudantes de de Broglie, é o primeiro a obter a álgebra $16 \times 16$ das matrizes de $\mathrm{DKP}^{3}$ [10]. Mas eram tempos de guerra, e o trabalho de Petiau só viria a público quando a Segunda Guerra Mundial chegasse ao fim [11].

Simultânea e completamente alheio ao trabalho de Petiau, Kemmer escreve as equações de segunda

\footnotetext{
${ }^{1}$ Em verdade, esta equação foi considerada pela primeira vez por Schrödinger para o estudo do espectro do átomo de hidrogênio. De fato, este estudo foi anterior à sua formulação da teoria não-relativística 6 .

${ }^{2}$ Pauli e Weisskopf 7], em 1934, interpretaram (6) como uma densidade de carga.

${ }^{3} \mathrm{Em}$ verdade, foi Géhéniau o precursor na decomposição desta álgebra em representações irredutíveis de 10 dimensões, 5 dimensões e uma representação trivial em 1 dimensão 9 .
} 
ordem de Proca como um conjunto de equações de primeira ordem acopladas. Faz o mesmo com a equação de Klein-Gordon-Fock. Kemmer então conjectura acerca da existência de uma forma matricial para seu sistema de equações acopladas, de forma que elas possuam representações irredutíveis, representando as partículas de spin 0 e de spin 1, mas não obtém sucesso em determinar a álgebra que tais matrizes devem satisfazer. Duffin, um matemático, tomou conhecimento das equações de Proca em um seminário dado por Kemmer e, simpático com sua abordagem em primeira ordem, desenvolve a álgebra tão desejada para a teoria 12 .

Embora não tenha sido um procedimento trivial obter a equação e álgebra de Duffin-Kemmer-Petiau, é possível se ter uma certa intuição de como obtêlas [13, 14: seja a equação de Klein-Gordon-Fock (em unidades naturais tais que $\hbar=c=1$ )

$$
\left(\partial_{\mu} \partial^{\mu}+m^{2}\right) \Phi(\vec{r}, t)=0
$$

em que $\Phi(\vec{r}, t)$ é uma função de onda que descreve uma partícula. É possível tornar esta equação de segunda ordem em uma equação de primeira ordem reescrevendo-a convenientemente como

$$
\frac{1}{m} g^{\mu \nu} \partial_{\mu} \partial_{\nu} \Phi(\vec{r}, t)+m \Phi(\vec{r}, t)=0
$$

e definindo

$$
\frac{1}{m} \partial_{\nu} \Phi(\vec{r}, t) \equiv \Phi_{\nu}(\vec{r}, t)
$$

em que $\nu=0,1,2,3$. De fato, este é o "preço a ser pago" pela redução da ordem da equação: o surgimento de novas variáveis. Aqui, estas novas variáveis são $\Phi_{\nu}(\vec{r}, t)$ e não têm significado físico. Assim, (11) se torna

$$
\partial^{\nu} \Phi_{\nu}(\vec{r}, t)+m \Phi(\vec{r}, t)=0
$$

Então, a equação de segunda ordem original (10) foi reduzida a um sistema de duas equações de primeira ordem, 12 e 13. Ao aplicar o operador diferencial $\partial_{\nu}$ à equação (13)

$$
\partial_{\nu} \partial^{\nu} \Phi_{\nu}(\vec{r}, t)+m^{2} \partial_{\nu} \Phi(\vec{r}, t)=0
$$

com (12), conduz a

$$
\left(\partial_{\nu} \partial^{\nu}+m^{2}\right) \Phi_{\nu}(\vec{r}, t)=0
$$

Portanto, uma equação de onda relativística em primeira ordem é tal que todos os componentes da função de onda, sejam eles físicos ou não-físicos (neste caso, $\Phi(\vec{r}, t)$ e $\Phi_{\nu}(\vec{r}, t)$, respectivamente) obedecem à equação de Klein-Gordon-Fock.

Umezawa 15 generaliza este procedimento: seja uma função de onda multicomponente $\Phi_{\mu}(x)$, em que $\mu=1,2, \ldots, n$, ou seja, $\Phi_{\mu}(x)$ é uma matriz coluna e $x$ é um quadrivetor com componentes $t \mathrm{e}$ $\vec{r}$. A equação relativística para esta função de onda tem a forma

$$
\Lambda_{\beta \mu}(\partial) \Phi_{\mu}(x)=0
$$

em que $\Lambda_{\beta \mu}(\partial)$ é um operador matricial. A condição de Klein-Gordon-Fock exige que (15) satisfaça a relação

$$
d_{\rho \beta}(\partial) \Lambda_{\beta \mu}(\partial)=\left(\square+m^{2}\right) \delta_{\rho \mu}
$$

em que $d(\partial)$ é um operador diferencial tal que

$$
d(\partial) \equiv\left[d_{\rho \beta}(\partial)\right]=\alpha+\alpha^{\nu} \partial_{\nu}+\alpha^{\nu \sigma} \partial_{\nu} \partial_{\sigma}+\ldots
$$

onde os coeficientes $\alpha, \alpha^{\nu}, \alpha^{\nu \sigma}, \ldots$ são matrizes de dimensão $n$ (em acordo com $\Phi_{\mu}(x)$ ).

Uma equação relativística como um conjunto de equações diferenciais em primeira ordem é escrita na forma

$$
\left(i \beta^{\mu} \partial_{\mu}-m I\right) \Phi(x)=0
$$

em que $\beta^{\mu}$ são certas matrizes de dimensão $n, I$ é a matriz identidade $n \times n$ e $\Phi(x)$ é uma matriz coluna também de dimensão $n$.

Facilmente se identifica

$$
\Lambda(\partial) \equiv\left[\Lambda_{\beta \mu}(\partial)\right]=\left(i \beta^{\mu} \partial_{\mu}-m I\right)
$$

e

$$
\Phi(x)=\left[\Phi_{\mu}(x)\right]
$$

o que deixa evidente que a quantidade $\Phi(x)$ é uma função de onda multicomponente. Considerando as particularizações:

\subsubsection{Caso I $d(\partial)=\alpha+\alpha^{\nu} \partial_{\nu}$}

Neste caso, 16 é tal que

$$
\left(\alpha+\alpha^{\nu} \partial_{\nu}\right)\left(i \beta^{\mu} \partial_{\mu}-m I\right)=\square+m^{2}
$$

ou seja,

$$
\begin{aligned}
& i \alpha \beta^{\mu} \partial_{\mu}-(\alpha I) m+i \alpha^{\nu} \beta^{\mu} \partial_{\mu} \partial_{\nu}-\left(\alpha^{\nu} I\right) m \partial_{\nu} \\
& =\left(\square+m^{2}\right) \\
& i\left(\alpha \beta^{\mu}\right) \partial_{\mu}-(\alpha I) m+\frac{i}{2}\left(\alpha^{\nu} \beta^{\mu}+\alpha^{\mu} \beta^{\nu}\right) \partial_{\mu} \partial_{\nu} \\
& -\left(\alpha^{\nu} I\right) m \partial_{\nu}=\left(\square+m^{2}\right)
\end{aligned}
$$

Revista Brasileira de Ensino de Física, vol. 38, nº 3, e3319, 2016 
para que esta igualdade seja verdadeira, as condições

$$
\begin{gathered}
i\left(\alpha \beta^{\mu}\right)-\left(\alpha^{\mu} I\right) m=0 \\
\frac{i}{2}\left(\alpha^{\nu} \beta^{\mu}+\alpha^{\mu} \beta^{\nu}\right)=g^{\mu \nu} \\
-(\alpha I) m=m^{2} I
\end{gathered}
$$

devem ser obedecidas. Estas condições conduzem a

$$
\begin{gathered}
\alpha=-m I \\
\alpha^{\mu}=-i \beta^{\mu}
\end{gathered}
$$

e, por fim

$$
\beta^{\nu} \beta^{\mu}+\beta^{\mu} \beta^{\nu}=2 g^{\mu \nu} .
$$

Portanto, identificando os termos de 18 com as condições acima, se obtém que

$$
\left(i \beta^{\mu} \partial_{\mu}-m\right) \Phi(x)=0
$$

e a função de onda multicomponente $\Phi(x)$ é reconhecida como o espinor $\psi(x)$ na renomeação de $\beta$ por $\gamma$

$$
\left(i \gamma^{\mu} \partial_{\mu}-m\right) \psi(x)=0
$$

$\mathrm{e}$

$$
\gamma^{\nu} \gamma^{\mu}+\gamma^{\mu} \gamma^{\nu}=2 g^{\mu \nu}
$$

que correspondem à equação de Dirac e sua álgebra 4

1.0.2. Caso II $d(\partial)=\alpha+\alpha^{\nu} \partial_{\nu}+\alpha^{\nu \sigma} \partial_{\nu} \partial_{\sigma}$

De maneira análoga ao caso anterior, a 16 agora se escreve como

$$
\left(\alpha+\alpha^{\nu} \partial_{\nu}+\alpha^{\nu \sigma} \partial_{\nu} \partial_{\sigma}\right)\left(i \beta^{\mu} \partial_{\mu}-m I\right)=\square+m^{2}
$$

ou seja,

$$
\begin{aligned}
& i\left(\alpha \beta^{\mu}\right) \partial_{\mu}-(\alpha I) m+\frac{i}{2}\left(\alpha^{\nu} \beta^{\mu}+\alpha^{\mu} \beta^{\nu}\right) \partial_{\mu} \partial_{\nu} \\
& -\left(\alpha^{\nu} I\right) m \partial_{\nu}
\end{aligned}
$$

$$
\begin{aligned}
& +\sum^{(P)}\left(i \alpha^{\nu \sigma} \beta^{\mu}\right) \partial_{\nu} \partial_{\sigma} \partial_{\mu}-\left(\alpha^{\nu \sigma} I\right) m \partial_{\nu} \partial_{\sigma} \\
& =\left(\square+m^{2}\right)
\end{aligned}
$$

em que $\sum(P)$ indica a soma sobre todas as permutações possíveis entre $\nu, \sigma$ e $\mu$ devido à simetria de $\partial_{\nu} \partial_{\sigma} \partial_{\mu}$. Portanto, para que esta igualdade seja satisfeita, as condições

$$
\begin{gathered}
i\left(\alpha \beta^{\mu}\right)-\left(\alpha^{\mu} I\right) m=0 \\
-(\alpha I) m=m^{2} I \\
\frac{i}{2}\left(\alpha^{\nu} \beta^{\mu}+\alpha^{\mu} \beta^{\nu}\right)-\alpha^{\mu \nu} m=g^{\mu \nu} \\
\sum(P)\left(i \alpha^{\nu \sigma} \beta^{\mu}\right)=0
\end{gathered}
$$

devem ser obedecidas. Estas condições conduzem a

$$
\begin{gathered}
\alpha=-m I \\
\alpha^{\mu}=-i \beta^{\mu} \\
\alpha^{\mu \nu}=-\frac{1}{m}\left[\delta^{\mu \nu}-\frac{1}{2}\left(\beta^{\mu} \beta^{\nu}+\beta^{\nu} \beta^{\mu}\right)\right]
\end{gathered}
$$

e

$$
\sum^{(P)}\left[\frac{1}{2}\left(\beta^{\nu} \beta^{\sigma} \beta^{\mu}+\beta^{\sigma} \beta^{\nu} \beta^{\mu}\right)-g^{\nu \sigma} \beta^{\mu}\right]=0 .
$$

Esta última relação, tomadas todas as permutações possíveis, conduz à álgebra ${ }^{5}$

$$
\beta^{\mu} \beta^{\nu} \beta^{\sigma}+\beta^{\sigma} \beta^{\nu} \beta^{\mu}=g^{\mu \nu} \beta^{\sigma}+g^{\sigma \nu} \beta^{\mu},
$$

e, identificando os termos de (15) com as condições supracitadas se determina o sistema de equações em primeira ordem

$$
i \beta^{\mu} \partial_{\mu} \psi-m \psi=0
$$

As equações 24 e sua álgebra 23ão as equações de Duffin-Kemmer-Petiau, doravante denominadas equações de DKP.

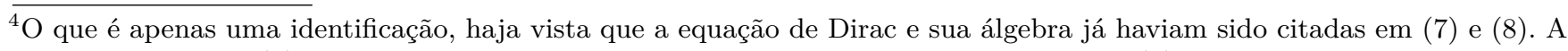
caracterização de $\Phi(x)$ com um espinor ocorre apenas com o estudo das matrizes $\gamma$ e de $\Phi(x)$ sob transformações de Lorentz. ${ }^{5}$ É ainda possível determinar esta álgebra partindo-se da ideia de de Broglie, ou seja, combinando dois léptons. Seja $\beta^{\mu}$ então escrita como

em que $\Gamma^{\mu}=\gamma^{\mu} \otimes 1$ e $\bar{\Gamma}^{\mu}=1 \otimes \gamma^{\mu}$. Com

$$
\beta^{\mu}=\frac{1}{2}\left(\Gamma^{\mu}+\bar{\Gamma}^{\mu}\right)
$$

e

$$
\left\{\Gamma^{\mu}, \Gamma^{\nu}\right\}=2 g^{\mu \nu}=\left\{\bar{\Gamma}^{\mu}, \bar{\Gamma}^{\nu}\right\}
$$$$
\left[\Gamma^{\mu}, \bar{\Gamma}^{\nu}\right]=0
$$

então reescrever a soma $\beta^{\mu} \beta^{\nu} \beta^{\sigma}+\beta^{\sigma} \beta^{\nu} \beta^{\nu}$ em termos dos $\Gamma$ e $\bar{\Gamma}$ conduz diretamente a 23 .
} 


\section{Propriedades gerais}

\subsection{A equação de DKP e a álgebra das matrizes $\beta^{\mu}$}

O formalismo de primeira ordem de DKP descreve partículas massivas de spin 0 (escalares) e spin 1 (vetoriais). A equação de DKP fundamental de tais partículas se expressa por

$$
i \beta^{\mu} \partial_{\mu} \psi-m \psi=0
$$

em que as matrizes $\beta^{\mu}$ satisfazem a álgebra

$$
\beta^{\mu} \beta^{\nu} \beta^{\sigma}+\beta^{\sigma} \beta^{\nu} \beta^{\mu}=g^{\mu \nu} \beta^{\sigma}+g^{\sigma \nu} \beta^{\mu}
$$

e o tensor métrico do espaço-tempo de Minkowski $g^{\mu \nu}$ tem assinatura $(+,-,-,-)$.

As matrizes $\beta^{\mu}$ têm dimensão 16 e constituem em uma representação redutível da álgebra (26). Esta álgebra admite somente três representações irredutíveis: uma representação irredutível em 10 dimensões (representando partículas de spin 1), 5 dimensões (representando partículas de spin 0) e uma representação trivial sem significado físico em 1 dimensão. O objeto $\psi$ é uma função de onda multicomponente: para partículas de spin 0 apresenta-se como uma função de onda de 5 componentes; para partículas de spin 1, como uma função de onda de 10 componentes.

Existe um conjunto de matrizes hermitianas $\eta^{\mu}$ que complementa as matrizes $\beta^{\mu}$, definidas por

$$
\eta^{\mu} \equiv 2 \beta^{\mu} \beta^{\mu}-g^{\mu \mu}
$$

de tal forma que as matrizes $\beta^{\mu}$ têm seu conjugado hermitiano através de

$$
\beta^{\mu \dagger}=\eta^{0} \beta^{\mu} \eta^{0}
$$

A equação de DKP conjugada é obtida tomando o conjugado hermitiano de 25

$$
-i\left(\partial_{\mu} \psi^{\dagger} \eta^{0}\right) \beta^{\mu}-m\left(\psi^{\dagger} \eta^{0}\right)=0
$$

definindo a função de onda adjunta $\bar{\psi}$ por

$$
\bar{\psi} \equiv \psi^{\dagger} \eta^{0}
$$

então, a equação de DKP conjugada é dada por

$$
i \partial_{\mu} \bar{\psi} \beta^{\mu}+m \bar{\psi}=0
$$

Uma prática manipulação matemática permite a obtenção da equação da continuidade na teoria de
DKP . Multiplicações apropriadas de $\bar{\psi}$ e $\psi$ em (25) e (30), respectivamente, seguida de sua soma, levam a

$$
\partial_{\mu}\left(\bar{\psi} \beta^{\mu} \psi\right)=0
$$

de tal forma que é possível definir a densidade de quadricorrente

$$
J^{\mu} \equiv \bar{\psi} \beta^{\mu} \psi
$$

e, portanto

$$
\partial_{\mu} J^{\mu}=0
$$

porém, o componente temporal da densidade de quadricorrente, $J^{0}$, não é positivo-definido, o que impede sua interpretação como uma densidade de probabilidade. Todavia $J^{0}$ pode ser interpretado como uma densidade de carga.

Por fim, multiplicar 25 por $\partial_{\alpha} \beta^{\alpha} \beta^{\nu}$ à esquerda leva a

$$
\partial^{\nu} \psi=\beta^{\alpha} \beta^{\nu} \partial_{\alpha} \psi
$$

de tal forma que a contração com $\partial_{\nu}$ conduz a

$$
\square \psi+m^{2} \psi=0
$$

mostrando que cada componente de $\psi$ satisfaz a condição de Klein-Gordon, como esperado para uma equação relativística.

A invariância da equação de Duffin-KemmerPetiau sob as transformações de Lorentz

$$
\begin{gathered}
x^{\mu \prime}=\Lambda_{\nu}^{\mu} x^{\nu} \\
\partial_{\mu}^{\prime}=\left(\Lambda^{-1}\right)_{\mu}^{\nu} \partial_{\nu} \\
\psi^{\prime}\left(x^{\prime}\right)=U(\Lambda) \psi(x) \\
m^{\prime}=m
\end{gathered}
$$

se expressa pela invariância da equação

$$
i \beta^{\mu \prime} \partial_{\mu}^{\prime} \psi^{\prime}\left(x^{\prime}\right)-m^{\prime} \psi^{\prime}\left(x^{\prime}\right)=0
$$

sob estas transformações. Para isso, é necessário determinar a forma explícita das transformações $U(\Lambda)$ e $\beta^{\mu \prime}$. Assim,

$$
i \beta^{\mu \prime}\left(\Lambda^{-1}\right)_{\mu}^{\nu} U(\Lambda) \partial_{\nu} \psi(x)-m U(\Lambda) \psi(x)=0
$$

aplicando a transformação inversa $U^{-1}(\Lambda)$ à esquerda

$i\left(U^{-1}(\Lambda) \beta^{\mu \prime}\left(\Lambda^{-1}\right)_{\mu}^{\nu} U(\Lambda)\right) \partial_{\nu} \psi(x)-m \psi(x)=0$ portanto, as matrizes $\beta$ têm de se transformar como

$$
U^{-1}(\Lambda) \beta^{\mu \prime}\left(\Lambda^{-1}\right)_{\mu}^{\nu} U(\Lambda)=\beta^{\nu}
$$

Revista Brasileira de Ensino de Física, vol. 38, nº 3, e3319, 2016 
ou seja

$$
U^{-1}(\Lambda) \beta^{\mu \prime} U(\Lambda)=\Lambda_{\nu}^{\mu} \beta^{\nu}
$$

uma vez determinada a forma explícita das matrizes $\beta$ sob transformações de Lorentz, o passo seguinte consiste em determinar $U(\Lambda)$. Seja a transformação infinitesimal

$$
U(\Lambda)=1+i \alpha \omega_{\mu \nu} S^{\mu \nu}+O\left(\omega^{2}\right)
$$

em que

$$
\Lambda_{\nu}^{\mu}=\delta_{\nu}^{\mu}+\omega_{\nu}^{\mu}+O\left(\omega^{2}\right), \quad \omega_{\mu \nu}=-\omega_{\nu \mu}
$$

esta transformação em (40) conduz a

$$
\begin{gathered}
\beta^{\mu \prime} U(\Lambda)=U(\Lambda) \Lambda_{\nu}^{\mu} \beta^{\nu} \\
\beta^{\mu \prime}+i \alpha \omega_{\rho \sigma} \beta^{\mu \prime} S^{\rho \sigma}=\beta^{\mu}+\omega^{\mu}{ }_{\nu} \beta^{\nu}+i \alpha \omega_{\rho \sigma} S^{\rho \sigma} \beta^{\mu}
\end{gathered}
$$

pela identidade nos polinômios em $\omega$, é possível inferir diretamente que

$$
\beta^{\mu \prime}=\beta^{\mu}
$$

e

$$
i \alpha \omega_{\rho \sigma}\left[\beta^{\mu}, S^{\rho \sigma}\right]=\omega^{\mu}{ }_{\nu} \beta^{\nu}
$$

que será usada para se determinar a forma explícita do gerador infinitesimal $S$ : simetrizando $\omega^{\mu}{ }_{\nu} \beta^{\nu}$

$$
\begin{aligned}
\omega_{\nu}^{\mu} \beta^{\nu} & =g^{\mu \sigma} \omega_{\sigma \nu} \beta^{\nu}=\frac{1}{2}\left(g^{\mu \sigma} \omega_{\sigma \nu} \beta^{\nu}-g^{\mu \sigma} \omega_{\nu \sigma} \beta^{\nu}\right) \\
& =\frac{1}{2}\left(g^{\mu \rho} \omega_{\rho \gamma} \beta^{\gamma}-g^{\mu \gamma} \omega_{\rho \gamma} \beta^{\rho}\right) \\
& =\frac{1}{2} \omega_{\rho \gamma}\left(g^{\mu \rho} \beta^{\gamma}-g^{\mu \gamma} \beta^{\rho}\right)
\end{aligned}
$$

então

$$
i \alpha \omega_{\rho \sigma}\left[\beta^{\mu}, S^{\rho \sigma}\right]=\frac{1}{2} \omega_{\rho \sigma}\left(g^{\mu \rho} \beta^{\sigma}-g^{\mu \sigma} \beta^{\rho}\right) .
$$

Da álgebra de DKP é possível inferir que

$$
g^{\mu \rho} \beta^{\sigma}-g^{\mu \sigma} \beta^{\rho}=-\left[\beta^{\mu},\left[\beta^{\rho}, \beta^{\sigma}\right]\right]
$$

assim

$$
i \alpha \omega_{\rho \sigma}\left[\beta^{\mu}, S^{\rho \sigma}\right]=-\frac{1}{2} \omega_{\rho \sigma}\left[\beta^{\mu},\left[\beta^{\rho}, \beta^{\sigma}\right]\right]
$$

portanto

$$
\alpha=-\frac{1}{2} \quad \text { e } \quad S^{\rho \sigma}=\left[\beta^{\rho}, \beta^{\sigma}\right]
$$

e a transformação $U(\Lambda)$ está determinada por

$$
U(\Lambda)=\exp \left(-\frac{1}{2} \omega_{\rho \sigma} S^{\rho \sigma}\right) .
$$

Uma representação escalar é tal que $U(\Lambda)=1$ (a matriz identidade), ao passo que uma representação vetorial se caracteriza por $U(\Lambda)=\Lambda$. A representação escalar caracteriza partículas de spin 0 , enquanto a representação vetorial, partículas de spin 1.

Por fim, a lei de transformação de $\bar{\psi}$ sob transformações de Lorentz é determinada a partir de

$$
\psi^{\prime}\left(x^{\prime}\right)=U(\Lambda) \psi(x)
$$

$\log 0$

$\bar{\psi}^{\prime}=\psi^{\dagger \prime} \eta^{0 \prime}=(U \psi)^{\dagger} \eta^{0}=\psi^{\dagger} U^{\dagger} \eta^{0}=\psi^{\dagger} \eta^{0}\left(\eta^{0} U^{\dagger} \eta^{0}\right)$

contudo

$$
U^{\dagger}=\exp \left(-\frac{1}{2} \omega_{\rho \sigma} S^{\dagger \rho \sigma}\right)
$$

em que

$$
\begin{aligned}
& S^{\dagger \rho \sigma}=\left(\beta^{\rho} \beta^{\sigma}\right)^{\dagger}-\left(\beta^{\sigma} \beta^{\rho}\right)^{\dagger}=\beta^{\sigma \dagger} \beta^{\rho \dagger} \\
& -\beta^{\rho \dagger} \beta^{\sigma \dagger}=\eta^{0} S^{\sigma \rho} \eta^{0}=-\eta^{0} S^{\rho \sigma} \eta^{0}
\end{aligned}
$$

então

$$
\begin{gathered}
\eta^{0} U^{\dagger} \eta^{0}=\eta^{0}\left(1-\frac{1}{2} \omega_{\rho \sigma} S^{\dagger \rho \sigma}+O\left(\omega^{2}\right)\right) \eta^{0} \\
=1+\frac{1}{2} \omega_{\rho \sigma} S^{\rho \sigma}+O\left(\omega^{2}\right)=\exp \left(\frac{1}{2} \omega_{\rho \sigma} S^{\rho \sigma}\right)=U^{-1}
\end{gathered}
$$

portanto

$$
\bar{\psi}^{\prime}=\psi^{\dagger} \eta^{0} U^{-1}=\bar{\psi} U^{-1}
$$

como esperado. Com a densidade de lagrangiana na forma

$$
\mathcal{L}=\frac{i}{2}\left(\bar{\psi} \beta^{\mu} \partial_{\mu} \psi-\partial_{\mu} \bar{\psi} \beta^{\mu} \psi\right)-m \bar{\psi} \psi
$$

que gera ambas equações de movimento, 25) e 30, é tal que, sob transformações de Lorentz

$$
\begin{aligned}
\mathcal{L}^{\prime} & =\frac{i}{2}\left(\bar{\psi}^{\prime} \beta^{\mu \prime} \partial^{\prime}{ }_{\mu} \psi^{\prime}-\partial^{\prime}{ }_{\mu} \bar{\psi}^{\prime} \beta^{\mu \prime} \psi^{\prime}\right)-m \bar{\psi}^{\prime} \psi^{\prime} \\
& =\frac{i}{2}\left\{\bar{\psi}\left[U^{-1} \beta^{\mu \prime} U\left(\Lambda^{-1}\right)_{\mu}^{\nu}\right] \partial_{\nu} \psi\right. \\
& \left.-\partial_{\nu} \bar{\psi}\left[\left(\Lambda^{-1}\right)_{\mu}^{\nu} U^{-1} \beta^{\mu \prime} U\right] \psi\right\}-m \bar{\psi} U^{-1} U \psi
\end{aligned}
$$

$\operatorname{com} 40$

$$
\begin{gathered}
\mathcal{L}^{\prime}=\frac{i}{2}\left\{\bar{\psi}\left[\Lambda_{\nu}^{\mu} \beta^{\nu}\left(\Lambda^{-1}\right)_{\mu}^{\nu}\right] \partial_{\nu} \psi\right. \\
\left.-\partial_{\nu} \bar{\psi}\left[\left(\Lambda^{-1}\right)_{\mu}^{\nu} \Lambda_{\nu}^{\mu} \beta^{\nu}\right] \psi\right\}-m \bar{\psi} \psi \\
\mathcal{L}^{\prime}=\frac{i}{2}\left(\bar{\psi} \beta^{\nu} \partial_{\nu} \psi-\partial_{\nu} \bar{\psi} \beta^{\nu} \psi\right)-m \bar{\psi} \psi=\mathcal{L}
\end{gathered}
$$

permanece invariante. 


\subsection{A equivalência entre o formalismo de DKP e os formalismos de Klein- Gordon-Fock e Proca: o caso livre}

\subsubsection{Os operadores de seleção}

Fujiwara [16], em 1953, desenvolve (baseado no trabalho de Peaslee [17]) duas duplas distintas de operadores que têm a prática propriedade de selecionar o setor de spin 0 ou o setor de spin 1 da teoria de DKP. Umezawa, em uma abordagem didática [15], explicita estes operadores e também suas propriedades.

\subsubsection{O setor de spin 0}

Seja um operador $P$, definido por

$$
P \equiv-\left(\beta^{0}\right)^{2}\left(\beta^{1}\right)^{2}\left(\beta^{2}\right)^{2}\left(\beta^{3}\right)^{2}
$$

em que $P^{2}=P$ e

$$
P^{\mu} \equiv P \beta^{\mu}
$$

é possível mostrar que $P$ e $P^{\mu}$ possuem as propriedades

$$
\begin{gathered}
P^{\mu} \beta^{\nu}=P g^{\mu \nu} \\
P S^{\mu \nu}=S^{\mu \nu} P=0 \\
P^{\mu} S^{\sigma \nu}=g^{\mu \sigma} P^{\nu}-g^{\mu \nu} P^{\sigma}
\end{gathered}
$$

Portanto, sob as transformações infinitesimais 44

$$
P U \psi=P \psi
$$

logo, $P \psi$ se transforma como um escalar. E é por isso que o operador $P$ seleciona o setor escalar da teoria de DKP. Analogamente,

$$
P^{\mu}(U \psi)=P^{\mu} \psi+\omega^{\mu}{ }_{\nu}\left(P^{\nu} \psi\right)
$$

ou seja, $P^{\mu}$ se transforma como um vetor.

A atuação do operador $P$ na equação de DKP (25) leva a

$$
i \partial_{\mu}\left(P^{\mu} \psi\right)-m(P \psi)=0
$$

analogamente, a atuação de $P^{\nu}$ em (25) fornece

$$
P^{\nu} \psi=\frac{i}{m} \partial^{\nu}(P \psi)
$$

substituindo (54) em (53)

$$
\left(\partial_{\mu} \partial^{\mu}+m^{2}\right)(P \psi)=0
$$

Portanto, elementos da matriz $P \psi$ são campos escalares de massa $m$ que obedecem a equação de Klein-Gordon-Fock.

Com apenas um grau de liberdade, da relação graus de liberdade e spin da partícula

$$
q=2 S+1
$$

é possível inferir que $P \psi$ descreve uma partícula de spin 0 .

Agora, denotando a função de onda multicomponente $\psi$ por

$$
\psi \equiv\left(\begin{array}{c}
\varphi^{\prime} \\
\psi^{0} \\
\psi^{1} \\
\psi^{2} \\
\psi^{3}
\end{array}\right)=\left(\begin{array}{c}
\varphi^{\prime} \\
\psi^{\nu}
\end{array}\right), \nu=0,1,2,3
$$

e com uma representação $5 \times 5$ para as matrizes $\beta^{\mu}$ (ver Apêndice A), a atuação do operador $P$ em $\psi$ fornece

$$
P \psi=\left(\begin{array}{c}
\varphi^{\prime} \\
0 \\
0 \\
0 \\
0
\end{array}\right)
$$

Logo, (54) é equivalente a

$$
P^{\nu} \psi=\frac{i}{m} \partial^{\nu} \varphi^{\prime}
$$

denotando

$$
\varphi^{\prime} \equiv \sqrt{m} \varphi
$$

então

$$
P^{\nu} \psi=\frac{i}{\sqrt{m}} \partial^{\nu} \varphi
$$

torna-se possível então definir

$$
\psi^{\nu} \equiv \frac{i}{\sqrt{m}} \partial^{\nu} \varphi
$$

e a função de onda multicomponente 57 pode ser reescrita como

$$
\psi=\left(\begin{array}{c}
\sqrt{m} \varphi \\
\frac{i}{\sqrt{m}} \partial^{\nu} \varphi
\end{array}\right)
$$


que é a forma física da função de onda de DKP do setor de spin 0. Logo,

$$
P \psi=\sqrt{m}\left(\begin{array}{c}
\varphi \\
0_{4 \times 1}
\end{array}\right)
$$

e

$$
P^{\nu} \psi=\frac{i}{\sqrt{m}}\left(\begin{array}{c}
\partial^{\nu} \varphi \\
0_{4 \times 1}
\end{array}\right)
$$

que claramente satisfaz

$$
\left(\partial_{\mu} \partial^{\mu}+m^{2}\right) \varphi=0
$$

\subsubsection{O setor de spin 1}

Agora, dado um operador $R^{\mu}$, definido por

$$
R^{\mu} \equiv\left(\beta^{1}\right)^{2}\left(\beta^{2}\right)^{2}\left(\beta^{3}\right)^{2}\left(\beta^{\mu} \beta^{0}-g^{\mu 0}\right)
$$

$\mathrm{e}$

$$
R^{\mu \nu} \equiv R^{\mu} \beta^{\nu}
$$

se obtém as propriedades

$$
\begin{gathered}
R^{\mu \nu}=-R^{\nu \mu} \\
S^{\nu \rho} R^{\mu}=0 \\
R^{\mu \nu} \beta^{\alpha}=R^{\mu} g^{\nu \alpha}-R^{\nu} g^{\mu \alpha} \\
R^{\mu} S^{\nu \rho}=g^{\mu \nu} R^{\rho}-g^{\mu \rho} R^{\nu} \\
R^{\mu \nu} S^{\rho \sigma}=g^{\nu \rho} R^{\mu \sigma}-g^{\mu \rho} R^{\nu \sigma}-g^{\nu \sigma} R^{\mu \rho}+g^{\mu \sigma} R^{\nu \rho}
\end{gathered}
$$

Tais operadores, sob transformações infinitesimais de Lorentz, 44), se comportam como

$$
R^{\mu} U \psi=R^{\mu} \psi+\omega^{\mu}{ }_{\nu}\left(R^{\nu} \psi\right)
$$

e

$$
R^{\mu \nu} U \psi=R^{\mu \nu} \psi+\omega_{\rho}^{\mu}\left(R^{\rho \nu} \psi\right)+\omega_{\rho}^{\nu}\left(R^{\nu \rho} \psi\right)
$$

mostrando que o operador $R^{\mu}$ seleciona os componentes de $\psi$ que se transformam como o componente $\mu$ de um quadrivetor, ao passo que $R^{\mu \nu}$ seleciona aqueles que se transformam como o componente $\mu \nu$ de um tensor de segunda ordem.
A aplicação do operador $R^{\mu}$ na equação de DKP (25) leva a

$$
\partial_{\nu} R^{\mu \nu} \psi=-i m R^{\mu} \psi
$$

enquanto $R^{\mu \nu}$ em (25) conduz a

$$
\partial^{\nu} R^{\mu} \psi-\partial^{\mu} R^{\nu} \psi=-i m R^{\mu \nu} \psi
$$

definindo

$$
U^{\mu \nu} \equiv \partial^{\mu}\left(R^{\nu} \psi\right)-\partial^{\nu}\left(R^{\mu} \psi\right)
$$

em que $U^{\mu \nu}=-U^{\nu \mu}$. Logo,

$$
R^{\mu \nu} \psi=-\frac{i}{m} U^{\mu \nu}
$$

substituindo (79) em 76

$$
\partial_{\nu} U^{\mu \nu}=m^{2} R^{\mu} \psi
$$

ou ainda

$$
\partial_{\nu} U^{\nu \mu}+m^{2} R^{\mu} \psi=0
$$

Explicitamente,

$$
\partial_{\nu}\left(\partial^{\nu} R^{\mu} \psi-\partial^{\mu} R^{\nu} \psi\right)+m^{2} R^{\mu} \psi=0
$$

que é a equação de Proca para uma partícula livre de spin 1. Mais ainda, aplicando $\partial_{\mu}$ à 80 obtemos

$$
\partial_{\mu} \partial_{\nu} \partial^{\nu} R^{\mu} \psi-\partial_{\mu} \partial^{\mu} \partial_{\nu} R^{\nu} \psi+m^{2} \partial_{\mu} R^{\mu} \psi=0
$$

ou seja,

$$
\partial_{\mu}\left(R^{\mu} \psi\right)=0
$$

aplicando 82) em (80)

$$
\left(\partial_{\nu} \partial^{\nu}+m^{2}\right) R^{\mu} \psi=0
$$

As equações 82 e 83 são equivalentes à equação de Proca 81). De 83 temos 4 graus de liberdade acessíves à partícula; no entanto 82 elimina um grau de liberdade, perfazendo 3 graus de liberdade. De (56), é possível inferir que este setor descreve uma partícula de spin 1.

Aplicando $R^{\mu}$ à função de onda multicomponente $\psi$, na representação das matrizes $\beta^{\mu}$ dadas por (ver Apêndice A) 


$$
R^{0} \psi=\left(\begin{array}{c}
\psi^{0} \\
0 \\
0 \\
0 \\
0 \\
0 \\
0 \\
0 \\
0 \\
0
\end{array}\right), \quad R^{1} \psi=\left(\begin{array}{c}
-\psi^{1} \\
0 \\
0 \\
0 \\
0 \\
0 \\
0 \\
0 \\
0 \\
0
\end{array}\right)
$$

Aparentemente, os componentes $\psi^{0}, \psi^{1}, \psi^{2}$ e $\psi^{3}$ são os componentes físicos da função de onda do setor de spin 1 da teoria de DKP. Todavia, 82 os vincula, perfazendo três componentes físicos. Os componentes restantes $\left(\psi^{4}, \psi^{5}, \psi^{6}, \psi^{7}, \psi^{8}\right.$ e $\left.\psi^{9}\right)$ são os componentes não-físicos do setor de spin 1 .

É importante ressaltar que o presente estudo mostrou explicitamente a equivalência entre formalismo de DKP e os formalismos de Klein-Gordon-Fock e Proca para o caso da partícula livre em nível clássico.

\subsection{A equivalência entre o formalismo de DKP e os formalismos de Klein-Gordon-Fock e Proca: o caso do acoplamento mínimo}

O setor escalar da teoria de DKP é selecionado com a aplicação do operador $P$ que, por sua vez, conduz à função de onda física (63). Esta expressão deve ser compatível com a invariância local de calibre:

$$
\psi^{\prime}(x)=e^{i e \alpha(x)} \psi(x)
$$

e

$$
\varphi^{\prime}(x)=e^{i e \alpha(x)} \varphi(x)
$$

É evidente que 63 não é invariante sob transformações locais de calibre. Tal fato é facilmente remediado com a usual prescrição $\partial_{\mu} \rightarrow D_{\mu}$, em que

$$
D_{\mu}=\partial_{\mu}+i A_{\mu}
$$

Desta feita, a função de onda multicomponente se escreve como

$$
\psi=\left(\begin{array}{c}
\sqrt{m} \varphi \\
i \\
\sqrt{m} D^{\nu} \varphi
\end{array}\right)
$$

compatível com a invariância local de calibre [19].

$$
R^{2} \psi=\left(\begin{array}{c}
-\psi^{2} \\
0 \\
0 \\
0 \\
0 \\
0 \\
0 \\
0 \\
0 \\
0
\end{array}\right), \quad R^{3} \psi=\left(\begin{array}{c}
-\psi^{3} \\
0 \\
0 \\
0 \\
0 \\
0 \\
0 \\
0 \\
0 \\
0
\end{array}\right)
$$

A analogia com o procedimento da seção anterior é completa. A equação de DKP com acoplamento vetorial mínimo é escrita como

$$
\left(i \beta^{\mu} \partial_{\mu}-m-\beta^{\mu} A_{\mu}\right) \psi=0
$$

ou ainda

$$
i \beta^{\mu} D_{\mu} \psi-m \psi=0
$$

O setor escalar da teoria de DKP é selecionado com a aplicação do operador $P$. Desta feita, aplicando o operador $P$ à equação 90

$$
i P \beta^{\mu} D_{\mu} \psi-m P \psi=0
$$

e, como $P \beta^{\mu}=P^{\mu}$,

$$
i D_{\mu} P^{\mu} \psi-m P \psi=0
$$

A atuação do operador $P^{\nu}$ em 90 conduz a

$$
i P^{\nu} \beta^{\mu} D_{\mu} \psi-m P^{\nu} \psi=0
$$

uma vez que $P^{\nu} \beta^{\mu}=g^{\mu \nu} P$,

$$
P^{\nu} \psi=\frac{i}{m} D^{\nu} P \psi
$$

Substituindo $(93)$ em 92

$$
\left(D_{\mu} D^{\mu}+m^{2}\right)(P \psi)=0
$$

e, uma vez que $P \psi$ determina o componente físico do espinor de DKP do setor escalar, é então nítido que o componente físico da função de onda multicomponente obedece à equação de Klein-GordonFock minimamente acoplada. Fazendo uso da forma explícita de $P \psi,(58)$, a equação 93 é equivalente a

$$
P^{\nu} \psi=\frac{i}{\sqrt{m}} D^{\nu} \varphi
$$

logo, os elementos de $P^{\nu} \psi$ são $\frac{i}{m}$ vezes a derivada covariante dos elementos de $P \psi$ correspondentes. 
O setor vetorial é selecionado com o a atuação do operador $R^{\nu}$ na equação 90 , obtendo

$$
i R^{\nu} \beta^{\mu} D_{\mu} \psi-m R^{\nu} \psi=0
$$

como $R^{\nu} \beta^{\mu}=R^{\nu \mu}$,

$$
i R^{\nu \mu} D_{\mu} \psi-m R^{\nu} \psi=0
$$

então

$$
D_{\mu}\left(R^{\nu \mu} \psi\right)+i m R^{\nu} \psi=0
$$

Atuar $R^{\nu \mu}$ em 90 leva a

$$
i R^{\nu \mu} \beta^{\alpha} D_{\alpha} \psi-m R^{\nu \mu} \psi=0
$$

e, com a identidade $R^{\nu \mu} \beta^{\alpha}=R^{\nu} g^{\mu \alpha}-R^{\mu} g^{\nu \alpha}$

$$
R^{\nu \mu} \psi=-\frac{i}{m}\left(D^{\nu} R^{\mu} \psi-D^{\mu} R^{\nu} \psi\right)
$$

substituindo (97) em 96

$$
D_{\mu}\left(D^{\mu} R^{\nu} \psi-D^{\nu} R^{\mu} \psi\right)+m^{2} R^{\nu} \psi=0
$$

Equivalente à equação de Proca minimamente acoplada. Sob as transformações locais de calibre (85) e (86), 98) é invariante, como deveriam ser.

A primeira vista, os resultados obtidos apresentam plena equivalência com os formalismos de KleinGordon e Proca. E, de fato, são. Porém, apenas ao fim dos anos 90 o problema do chamado termo anômalo na teoria de DKP foi resolvido por Nowakowski [18] (também em [19]) em um procedimento até certo ponto análogo ao feito aqui. Este tal termo anômalo impedia que os formalismos fossem equivalentes. Todavia este termo não apresenta significado físico e é por isso que a aplicação dos operadores $P$ e $R^{\mu}$, que selecionam apenas os componentes físicos das funções de onda, fazem com que este termo desapareça. É importante ressaltar também que o termo anômalo já era conhecido por Kemmer em seu trabalho original. De fato, Kemmer é o primeiro a perceber que este termo não tem uma interpretação física, tampouco algum análogo na teoria de Dirac. Explicitamente, é possível perceber o termo anômalo em um simples procedimento: contraindo a equação de DKP minimamente acoplada 90 com o termo $D_{\alpha} \beta^{\alpha} \beta^{\nu}$ à esquerda leva a

$$
i \beta^{\alpha} \beta^{\nu} \beta^{\mu} D_{\alpha} D_{\mu} \psi-m \beta^{\alpha} \beta^{\nu} D_{\alpha} \psi=0
$$

que pode ser reescrita como

$$
D^{\nu} \psi=\beta^{\mu} \beta^{\nu} D_{\mu} \psi+\frac{e}{2 m} F_{\alpha \mu}\left(\beta^{\mu} \beta^{\nu} \beta^{\alpha}+\beta^{\mu} g^{\nu \alpha}\right) \psi
$$

Agora, contraindo esta expressão com $D_{\nu}$

$$
\begin{aligned}
& D_{\nu} D^{\nu} \psi=\beta^{\mu} \beta^{\nu} D_{\nu} D_{\mu} \psi \\
& +\frac{e}{2 m} F_{\alpha \mu}\left(\beta^{\mu} \beta^{\nu} \beta^{\alpha}+\beta^{\mu} g^{\nu \alpha}\right) D_{\nu} \psi
\end{aligned}
$$

ou ainda

$$
\begin{aligned}
& D_{\nu} D^{\nu} \psi+m^{2} \psi-\frac{i}{2} e F_{\mu \nu} S^{\mu \nu} \psi \\
& -\frac{e}{2 m}\left(\beta^{\mu} \beta^{\nu} \beta^{\alpha}+\beta^{\mu} g^{\nu \alpha}\right) D_{\nu}\left(F_{\alpha \mu} \psi\right) \\
& =0
\end{aligned}
$$

em que $\left[D_{\mu}, D_{\nu}\right]=-i e F_{\mu \nu}$. O termo proporcional a $e F_{\mu \nu} S^{\mu \nu} \psi$ descreve a interação do campo eletromagnético externo com os momentos elétrico e magnético da partícula. O termo anômalo é aquele proporcional a $\frac{e}{2 m}$.

Para o setor de spin 0 da teoria de DKP, aplicar o operador $P$ na equação 102 conduz a

$$
\begin{aligned}
& D_{\nu} D^{\nu}(P \psi)+m^{2}(P \psi)-\frac{i}{2} e F_{\mu \nu} P S^{\mu \nu} \psi \\
& -\frac{e}{2 m} P\left(\beta^{\mu} \beta^{\nu} \beta^{\alpha}+\beta^{\mu} g^{\nu \alpha}\right) D_{\nu}\left(F_{\alpha \mu} \psi\right) \\
& =0
\end{aligned}
$$

que, por sua vez (e usando as propriedades 48, (49) e (50), leva a

$$
\begin{aligned}
& D_{\nu} D^{\nu}(P \psi)+m^{2}(P \psi) \\
& -\frac{e}{2 m}\left(g^{\mu \nu} P^{\alpha}+P^{\mu} g^{\nu \alpha}\right) D_{\nu}\left(F_{\alpha \mu} \psi\right) \\
& =0
\end{aligned}
$$

portanto

$$
D_{\nu} D^{\nu}(P \psi)+m^{2}(P \psi)=0
$$

idêntico ao obtido em (94).

É nítido que o operador que seleciona o setor de spin 0 da teoria de DKP anula o termo anômalo, uma vez que este termo não tem um significado físico. Portanto, o operador $P$ de fato seleciona os componentes físicos do setor de spin 0 da teoria de DKP. Em um procedimento análogo é possível também mostrar que o mesmo acontece no setor de spin 1 da teoria de DKP. 


\section{O problema com o limite $m=0$ na teoria de DKP}

É natural uma extensão para $m=0$ na teoria de DKP com a expectativa de que o setor vetorial reproduza as equações de Maxwell. Todavia, esta construção está intrinsecamente associada à uma simetria local de calibre e, de fato, não é trivial. Para que o problema se torne nítido, tome-se ingenuamente $m=0$, por exemplo, em 63

$$
\psi=\left(\begin{array}{c}
\sqrt{m} \varphi \\
\frac{i}{\sqrt{m}} \partial^{\nu} \varphi
\end{array}\right)
$$

isso conduz a resultados desastrosos para o componente físico da função de onda multicomponente de DKP: ele simplesmente seria zero!, e os componentes não-físicos seriam todos infinitos.

De fato, Harish-Chandra 20, em 1946, aborda o problema em um estudo sistemático do limite $m=0$. Uma abordagem didática deste trabalho será realizada aqui, com a análise da lagrangiana proposta por Harish-Chandra,

$$
\mathcal{L}=i \bar{\psi} \gamma \beta^{\alpha}\left(\partial_{\alpha} \psi\right)-i\left(\partial_{\alpha} \bar{\psi}\right) \beta^{\alpha} \gamma \psi-\bar{\psi} \gamma \psi
$$

em que a álgebra de DKP foi então aqui complementada com a adição de uma matriz $\gamma$. Para conhecer as propriedades desta nova matriz $\gamma$, é interessante valer-se do fato de que a lagrangiana deve ser hermitiana. Então

$$
\begin{aligned}
& \mathcal{L}^{\dagger}=\left[i \psi^{\dagger} \eta^{0} \gamma \beta^{\alpha}\left(\partial_{\alpha} \psi\right)\right]^{\dagger}-\left[i\left(\partial_{\alpha} \psi^{\dagger}\right) \eta^{0} \beta^{\alpha} \gamma \psi\right]^{\dagger} \\
& -\left[\psi^{\dagger} \eta^{0} \gamma \psi\right]^{\dagger} \\
& \mathcal{L}^{\dagger}=i\left(\partial_{\alpha} \psi^{\dagger}\right) \beta^{\alpha \dagger} \gamma^{\dagger} \eta^{0 \dagger} \psi-i \psi^{\dagger} \gamma^{\dagger} \beta^{\alpha \dagger} \eta^{0 \dagger}\left(\partial_{\alpha} \psi\right) \\
& -\psi^{\dagger} \gamma^{\dagger} \eta^{0 \dagger} \psi
\end{aligned}
$$

como $\eta^{0 \dagger}=\eta^{0}, \beta^{\alpha \dagger}=\eta^{0} \beta^{\alpha} \eta^{0}$ e $\left(\eta^{0}\right)^{2}=1$, então

$$
\begin{aligned}
& \mathcal{L}^{\dagger}=i \partial_{\alpha} \psi^{\dagger} \eta^{0} \beta^{\alpha} \eta^{0} \gamma^{\dagger} \eta^{0} \psi \\
& -i \psi^{\dagger} \eta^{0} \eta^{0} \gamma^{\dagger} \eta^{0} \beta^{\alpha}\left(\partial_{\alpha} \psi\right)-\psi^{\dagger} \eta^{0} \eta^{0} \gamma^{\dagger} \eta^{0} \psi
\end{aligned}
$$

por fim

$\mathcal{L}^{\dagger}=i\left(\partial_{\alpha} \bar{\psi}\right) \beta^{\alpha} \eta^{0} \gamma^{\dagger} \eta^{0} \psi-i \bar{\psi} \eta^{0} \gamma^{\dagger} \eta^{0} \beta^{\alpha}\left(\partial_{\alpha} \psi\right)-\bar{\psi} \eta^{0} \gamma^{\dagger} \eta^{0}$

o que implica em

$$
\gamma^{\dagger}=\gamma
$$

e

$$
\left[\eta^{0}, \gamma\right]=0
$$

$\mathrm{E}$ as equações de movimento para a lagrangiana de Harish-Chandra são obtidas pelas equações de Euler-Lagrange

$$
\begin{array}{r}
i\left(\gamma \beta^{\alpha}+\beta^{\alpha} \gamma\right)\left(\partial_{\alpha} \psi\right)-\gamma \psi=0 \\
i\left(\partial_{\alpha} \bar{\psi}\right)\left(\gamma \beta^{\alpha}+\beta^{\alpha} \gamma\right)+\bar{\psi} \gamma=0
\end{array}
$$

contudo, se

$$
\gamma \beta^{\alpha}+\beta^{\alpha} \gamma=\beta^{\alpha}
$$

o que é consistente ao se levar em conta que a hermiticidade desta expressão se expressa através de

$$
\begin{gathered}
\beta^{\alpha \dagger} \gamma^{\dagger}+\gamma^{\dagger} \beta^{\alpha \dagger}=\beta^{\alpha \dagger} \\
\eta^{0} \beta^{\alpha} \eta^{0} \gamma^{\dagger}+\gamma^{\dagger} \eta^{0} \beta^{\alpha} \eta^{0}=\eta^{0} \beta^{\alpha} \eta^{0}
\end{gathered}
$$

ou ainda

$$
\beta^{\alpha} \eta^{0} \gamma^{\dagger} \eta^{0}+\eta^{0} \gamma^{\dagger} \eta^{0} \beta^{\alpha}=\beta^{\alpha}
$$

garante a hermiticidade de 112 se obedecer às mesmas relações (108) e (109), relações que tornam a lagrangiana hermitiana. Portanto, $(112)$ é válida e as equações de Euler-Lagrange (110) e (111) se reescrevem como

$$
\begin{aligned}
& i \beta^{\alpha} \partial_{\alpha} \psi-\gamma \psi=0 \\
& i \partial_{\alpha} \bar{\psi} \beta^{\alpha}+\bar{\psi} \gamma=0
\end{aligned}
$$

que é a equação para o campo de DKP e sua equação adjunta, respectivamente, para o caso de massa nula.

A simetria local de calibre corresponde aqui às seguintes transformações infinitesimais

$$
\delta \psi(x)=(1-\gamma) \Phi(x)
$$

$\mathrm{e}$

$$
\delta \bar{\psi}(x)=\bar{\Phi}(x)(1-\gamma)
$$

e, embora esses parâmetros infinitesimais da transformação de calibre sejam dependentes do ponto, o processo de transformação dos campos ocorre a ponto fixo, uma vez que atua somente na forma do łampo, não em suas coordenadas. Logo, o princípio da invariância é válido, e a variação da forma do campo é igual à variação total do campo.

Assim, a variação da ação se escreve como 


$$
\begin{aligned}
\delta S= & \int_{\Omega} d x\left[i \delta \bar{\psi}(x) \gamma \beta^{\alpha}\left(\partial_{\alpha} \psi\right)+i \bar{\psi} \gamma \beta^{\alpha}\left(\partial_{\alpha} \delta \psi(x)\right)-i\left(\partial_{\alpha} \delta \bar{\psi}(x)\right) \beta^{\alpha} \gamma \psi\right. \\
& \left.-i\left(\partial_{\alpha} \bar{\psi}\right) \beta^{\alpha} \gamma \delta \psi(x)-\delta \bar{\psi}(x) \gamma \psi-\bar{\psi} \gamma \delta \psi(x)\right]
\end{aligned}
$$

em que a medida de integração é invariante. Portanto

$$
\begin{aligned}
\delta S= & \int_{\Omega} d x\left[i \bar{\Phi}(x)(1-\gamma) \gamma \beta^{\alpha}\left(\partial_{\alpha} \psi\right)+i \bar{\psi} \gamma \beta^{\alpha}(1-\gamma)\left(\partial_{\alpha} \Phi(x)\right)-i\left(\partial_{\alpha} \bar{\Phi}(x)\right)(1-\gamma) \beta^{\alpha} \gamma \psi\right. \\
& \left.-i\left(\partial_{\alpha} \bar{\psi}\right) \beta^{\alpha} \gamma(1-\gamma) \Phi(x)-\bar{\Phi}(x)(1-\gamma) \gamma \psi-\bar{\psi} \gamma(1-\gamma) \Phi(x)\right]
\end{aligned}
$$

que, com as equações de movimento, se torna

$$
\begin{aligned}
\delta S= & \int_{\Omega} d x\left[i \bar{\psi}\left(\beta^{\alpha}-\beta^{\alpha} \gamma\right)(1-\gamma)\left(\partial_{\alpha} \Phi(x)\right)-i\left(\partial_{\alpha} \bar{\Phi}(x)\right)(1-\gamma)\left(\beta^{\alpha}-\gamma \beta^{\alpha}\right) \psi\right. \\
& \left.+\bar{\psi}\left(\gamma^{2}-\gamma\right)(1-\gamma) \Phi(x)+\bar{\Phi}(x)(1-\gamma)\left(\gamma^{2}-\gamma\right) \psi\right]
\end{aligned}
$$

ou seja,

$$
\begin{aligned}
\delta S= & \int_{\Omega} d x\left[i \bar{\psi} \beta^{\alpha}(1-\gamma)\left(\partial_{\alpha} \Phi(x)\right)-i\left(\partial_{\alpha} \bar{\Phi}(x)\right)(1-\gamma) \beta^{\alpha} \psi+i \bar{\psi} \beta^{\alpha} \gamma\left(\gamma^{2}-\gamma\right)\left(\partial_{\alpha} \Phi(x)\right)\right. \\
& \left.+\bar{\psi}\left(\gamma^{2}-\gamma\right)(1-\gamma) \Phi(x)-i\left(\partial_{\alpha} \bar{\Phi}(x)\right)\left(\gamma^{2}-\gamma\right) \beta^{\alpha} \psi+\bar{\Phi}(x)(1-\gamma)\left(\gamma^{2}-\gamma\right) \psi\right]
\end{aligned}
$$

Portanto, as condições

$$
i \beta^{\alpha}(1-\gamma)\left(\partial_{\alpha} \Phi(x)\right)=0
$$

$\mathrm{e}$

$$
i\left(\partial_{\alpha} \bar{\Phi}(x)\right)(1-\gamma) \beta^{\alpha}=0
$$

que correspondem às condições de calibre, além da condição

$$
\gamma^{2}-\gamma=0
$$

sobre a matriz $\gamma$, haja vista que $\gamma \neq 1$ necessariamente, são condições que devem ser satisfeitas para que a variação da ação seja nula.

\subsubsection{O setor de spin 0}

Para o setor de spin 0, a abordagem mais prática é fazer o uso dos projetores (46) e (47) diretamente na equação de movimento (113). Porém, antes de efetuar estes cálculos é necessário que se determine as relações entre $P, P^{\mu}$ e $\gamma$.

De 1112 é possível reescrever

$$
\begin{gathered}
\beta^{\alpha} \gamma=\beta^{\alpha}-\gamma \beta^{\alpha} \\
\left(\beta^{\alpha}\right)^{2} \gamma=\left(\beta^{\alpha}\right)^{2}-\left(\beta^{\alpha} \gamma\right) \beta^{\alpha} \\
\left(\beta^{\alpha}\right)^{2} \gamma=\left(\beta^{\alpha}\right)^{2}-\left(\beta^{\alpha}-\gamma \beta^{\alpha}\right) \beta^{\alpha} \\
\left(\beta^{\alpha}\right)^{2} \gamma=\left(\beta^{\alpha}\right)^{2}-\left(\beta^{\alpha}\right)^{2}+\gamma\left(\beta^{\alpha}\right)^{2}
\end{gathered}
$$

$\log$,

$$
\left(\beta^{\alpha}\right)^{2} \gamma=\gamma\left(\beta^{\alpha}\right)^{2}
$$

o que implica em

$$
P \gamma=-\left(\beta^{0}\right)^{2}\left(\beta^{1}\right)^{2}\left(\beta^{2}\right)^{2}\left(\beta^{3}\right)^{2} \gamma=\gamma P
$$

desse modo,

$$
P \gamma=\gamma P
$$

e, aplicando $P$ em 112

$$
\begin{gathered}
P \gamma \beta^{\alpha}+P \beta^{\alpha} \gamma=P \beta^{\alpha} \\
\gamma P \beta^{\alpha}+P^{\alpha} \gamma=P^{\alpha}
\end{gathered}
$$

assim,

$$
\gamma P^{\alpha}+P^{\alpha} \gamma=P^{\alpha}
$$

Agora, aplicando $P$ e $P^{\mu}$ à equação de movimento (113) conduz a

$$
i \partial_{\mu}\left(P^{\mu} \psi\right)-\gamma(P \psi)=0
$$


e

$$
i \partial^{\mu}(P \psi)-P^{\mu} \gamma \psi=0
$$

respectivamente, o que leva a

$$
\partial_{\mu} \partial^{\mu}(P \psi)=\left(\gamma^{2}-\gamma\right) P \psi
$$

o que, de 119 , resulta em

$$
\partial_{\mu} \partial^{\mu}(P \psi)=0
$$

que é uma equação do tipo Klein-Gordon-Fock com massa nula. Já a transformação infinitesimal (115) toma a forma

$$
P \psi^{\prime}(x)=P \psi(x)+(1-\gamma) P \Phi(x)
$$

e

$$
P^{\mu} \psi^{\prime}(x)=P^{\mu} \psi(x)+P^{\mu}(1-\gamma) \Phi(x)
$$

por fim, a condição de calibre (117) se estabelece por

$$
\begin{gathered}
i P^{\mu}(1-\gamma)\left(\partial_{\mu} \Phi(x)\right)=0 \\
i(1-\gamma)\left(\partial^{\mu} P \Phi(x)\right)=0
\end{gathered}
$$

Os resultados até então obtidos são independentes de uma representação para as matrizes $\beta$ e $\gamma$. Todavia, torna-se agora necessário recorrer a uma representação explícita para tais matrizes para que seja possível depreender as consequências das transformações de calibre. Sendo assim, com 173a e com a matriz $\gamma$ escrita da forma mais geral que satisfaz a condição 112 dada por

$$
\gamma=\operatorname{diag}(\lambda, 1-\lambda, 1-\lambda, 1-\lambda, 1-\lambda)
$$

em que $\lambda \in \mathbb{C}$ é um parâmetro a ser determinado pela invariância de calibre.

Nesta representação

$$
\psi=\left(\begin{array}{c}
\varphi \\
\psi^{\alpha}
\end{array}\right)
$$

e

$$
P \psi=\left(\begin{array}{c}
\varphi \\
{[0]_{4 \times 1}}
\end{array}\right)
$$

e

$$
P \gamma \psi=\left(\begin{array}{c}
\lambda \varphi \\
{[0]_{4 \times 1}}
\end{array}\right)
$$

e

$$
P^{\alpha} \psi=\left(\begin{array}{c}
\psi^{\alpha} \\
{[0]_{4 \times 1}}
\end{array}\right)
$$

por fim

$$
P^{\alpha} \gamma \psi=\left(\begin{array}{c}
(1-\lambda) \psi^{\alpha} \\
{[0]_{4 \times 1}}
\end{array}\right)
$$

Agora, sujeitando a matriz $\gamma$ à condição 119 resulta que o parâmetro $\lambda$ satisfaz a equação $\lambda^{2}-\lambda=0$, ou seja, os valores possíveis para $\lambda$ são 0 e 1 .

As equações de movimento 122 e 123 nesta representação se escrevem como

$$
i \partial_{\alpha} \psi^{\alpha}=\lambda \varphi
$$

e

$$
i \partial^{\alpha} \varphi=(1-\lambda) \psi^{\alpha}
$$

respectivamente. Estas equações implicam em

$$
\partial_{\alpha} \partial^{\alpha} \varphi+\lambda(1-\lambda) \varphi=0
$$

que, independente dos valores possíveis para $\lambda$, conduz à

$$
\partial_{\alpha} \partial^{\alpha} \varphi=0
$$

ou seja, o componente físico da função de onda obedece a uma equação de Klein-Gordon-Fock com massa nula, como esperado.

Denotando $\Phi=\left(\varphi_{\Phi}, \phi^{0}, \phi^{1}, \phi^{2}, \phi^{3}\right)^{T}$, a transformação infinitesimal (125) se escreve como

$$
\varphi^{\prime}=\varphi+(1-\lambda) \varphi_{\Phi}
$$

que é a transformação do componente físico do campo de DKP. Para $\lambda=0$, essa transformação é uma transformação global de calibre, uma vez que $\varphi_{\Phi}$ é constante. Para $\lambda=1$, esta transformação corresponde a um campo constante, chamado de campo topológico, uma vez que não possui graus de liberdade propagantes.

Já a transformação infinitesimal (126) conduz à transformação

$$
\psi^{\alpha}(x)=\psi^{\alpha}(x)+\lambda \phi^{\alpha}(x)
$$

que se dará apenas entre os componentes não-físicos da função de onda multicomponente de DKP.

Por fim, as condições de calibre (127) e (128) são então obtidas, respectivamente, por

$$
\lambda \partial_{\mu} \phi^{\mu}=0
$$

que, para $\lambda=1$ vincula as componentes não-físicas do campo de DKP, e

$$
(1-\lambda) \partial^{\mu} \varphi_{\Phi}=0
$$


que, para $\lambda=0$, assegura que o campo $\varphi_{\Phi}$ é constante.

Para o caso em que $\lambda=0$, a expressão para a lagrangiana de Harish-Chandra (107) conduz à lagrangiana do tipo Klein-Gordon-Fock com massa nula

$$
\mathcal{L}_{s=0}=\partial^{\mu} \varphi^{*} \partial_{\mu} \varphi
$$

\subsubsection{O setor de $\operatorname{spin} 1$}

Para o setor de spin 1, a abordagem é análoga: os projetores (67) e (68) obedecem a

$$
\gamma R^{\mu}=R^{\mu} \gamma
$$

e

$$
\gamma R^{\mu \nu}+R^{\mu \nu} \gamma=R^{\mu \nu}
$$

Assim, aplicando $R^{\mu}$ e $R^{\mu \nu}$ à equação de movimento (113) conduz a

$$
i \partial_{\alpha} R^{\mu \alpha} \psi-\gamma R^{\mu} \psi=0
$$

e

$$
i \partial^{\nu} R^{\mu} \psi-i \partial^{\mu} R^{\nu} \psi-R^{\mu \nu} \gamma \psi=0
$$

respectivamente; estas duas equações podem ser reescritas em uma única equação, a saber

$$
-\partial_{\alpha} \partial^{\alpha} R^{\mu} \psi+\partial_{\alpha} \partial^{\mu} R^{\alpha} \psi+\left(\gamma^{2}-\gamma\right) R^{\mu} \psi=0
$$

e, com 119 resulta em

$$
\partial_{\alpha}\left[\partial^{\mu}\left(R^{\alpha} \psi\right)-\partial^{\alpha}\left(R^{\mu} \psi\right)\right]=0
$$

ou seja, uma equação do tipo Proca com massa nula. A transformação infinitesimal 115 tem agora as formas

$$
R^{\mu} \psi^{\prime}(x)=R^{\mu} \psi(x)+(1-\gamma) R^{\mu} \Phi(x)
$$

e

$$
R^{\mu \nu} \psi^{\prime}(x)=R^{\mu \nu} \psi(x)+R^{\mu \nu}(1-\gamma) \Phi(x)
$$

e as condições de calibre (117) e (118) são dadas por

$$
R^{\mu \alpha}(1-\gamma)\left(\partial_{\alpha} \Phi(x)\right)=0
$$

$\mathrm{e}$

$$
(1-\gamma) \partial^{\nu}\left(R^{\mu} \Phi(x)\right)-(1-\gamma) \partial^{\mu}\left(R^{\nu} \Phi(x)\right)=0
$$

Com uma representação para as matrizes $\beta$ dada por 175a e com a matriz $\gamma$ escrita da forma mais geral que satisfaz a condição 112 para o setor de spin 1 dada por

$$
\gamma=\operatorname{diag}(\lambda, \lambda, \lambda, \lambda, 1-\lambda, 1-\lambda, 1-\lambda, 1-\lambda, 1-\lambda, 1-\lambda)
$$

novamente, $\lambda \in \mathbb{C}$ e será determinado pela invariância de calibre.

Nesta representação

$$
\psi=\left(\begin{array}{c}
{\left[\psi^{\mu}\right]_{4 \times 1}} \\
{\left[\psi^{\mu \nu}\right]_{6 \times 1}}
\end{array}\right)
$$

e

$$
R^{\mu} \psi=\left(\begin{array}{c}
\psi^{\mu} \\
{[0]_{9 \times 1}}
\end{array}\right)
$$

e

$$
R^{\mu} \gamma \psi=\left(\begin{array}{c}
\lambda \psi^{\mu} \\
{[0]_{9 \times 1}}
\end{array}\right)
$$

e

$$
R^{\mu \nu} \psi=\left(\begin{array}{c}
\psi^{\mu \nu} \\
{[0]_{9 \times 1}}
\end{array}\right)
$$

por fim

$$
R^{\mu \nu} \gamma \psi=\left(\begin{array}{c}
(1-\lambda) \psi^{\mu \nu} \\
{[0]_{9 \times 1}}
\end{array}\right)
$$

O parâmetro $\lambda$ satisfaz a equação $\lambda^{2}-\lambda=0$, ou seja, os valores possíveis para $\lambda$ são 0 e 1 . Portanto, as equações de movimento 140 e 141) agora se tornam

$$
i \partial_{\alpha} \psi^{\mu \alpha}=\lambda \psi^{\mu}
$$

e

$$
i \partial^{\nu} \psi^{\mu}-i \partial^{\mu} \psi^{\nu}-(1-\lambda) \psi^{\mu \nu}=0
$$

que, combinadas, conduzem a

$$
\partial_{\alpha}\left[\partial^{\alpha} \psi^{\mu}-\partial^{\mu} \psi^{\alpha}\right]=-\lambda(1-\lambda) \psi^{\mu}
$$

daqui se observa que independente do valor de $\lambda$ admissíveis, se mantém

$$
\partial_{\alpha}\left[\partial^{\alpha} \psi^{\mu}-\partial^{\mu} \psi^{\alpha}\right]=0
$$


com a condição de normalização

$$
\psi^{\mu}=\frac{1}{\sqrt{2}} A^{\mu}
$$

então

$$
\partial_{\alpha}\left[\partial^{\alpha} A^{\mu}-\partial^{\mu} A^{\alpha}\right]=0
$$

definindo o tensor eletromagnético $F^{\mu \nu} \equiv \partial^{\mu} A^{\nu}-$ $\partial^{\nu} A^{\mu}$, obtemos diretamente de 155 as equações de Maxwell

$$
\partial_{\mu} F^{\mu \nu}=0
$$

e também, para o quadrivetor $A^{\mu}$

$$
\square A^{\mu}-\partial^{\mu}\left(\partial_{\alpha} A^{\alpha}\right)=0
$$

que, satisfazendo a condição de Lorenz $\partial_{\alpha} A^{\alpha}=0$, conduz a

$$
\square A^{\mu}=0
$$

Seja a representação

$$
\Phi=\left(\begin{array}{c}
{\left[\phi^{\mu}\right]_{4 \times 1}} \\
{\left[\phi^{\mu \nu}\right]_{6 \times 1}}
\end{array}\right)
$$

as condições de calibre (145) e 146 se tornam

$$
\lambda \partial_{\alpha} \phi^{\mu \alpha}=0
$$

e

$$
(\lambda-1)\left[\partial^{\mu} \phi^{\nu}-\partial^{\nu} \phi^{\mu}\right]=0
$$

as transformações infinitesimais (143) e (144) se escrevem como

$$
\psi^{\mu}=\psi^{\mu}+(1-\lambda) \phi^{\mu}
$$

que, para $\lambda=0$ se reverte em

$$
\psi^{\mu}=\psi^{\mu}+\phi^{\mu}
$$

para ser ainda mais familiar, tomando

$$
\phi^{\mu}=\partial^{\mu} \Lambda(x)
$$

em que $\Lambda(x)$ é uma função escalar arbitrária. Assim, a transformação infinitesimal (163), para $\lambda=0$ se escreve como

$$
\psi^{\mu}=\psi^{\mu}+\partial^{\mu} \Lambda(x)
$$

que também satisfaz (162)

$$
\left[\partial^{\mu} \partial^{\nu} \Lambda(x)-\partial^{\nu} \partial^{\mu} \Lambda(x)\right]=0
$$

como deveria ser para que o tensor eletromagnético $F^{\mu \nu}$ seja invariante por (165).

Para o caso $\lambda=1$, a transformação infinitesimal (163) se reverte em

$$
\psi^{\mu}=\psi^{\mu}
$$

também relacionado a um campo topológico. Por fim, com a condição de normalização 156 na expressão para a lagrangiana de Harish-Chandra (107) conduz à lagrangiana de Maxwell

$$
\mathcal{L}_{s=1}=-\frac{1}{4} F_{\mu \nu} F^{\mu \nu}
$$

como esperado.

\subsection{As interações na teoria de DKP}

A equação de DKP com a interação mais geral possível é escrita como

$$
\left(i \beta^{\mu} \partial_{\mu}-m-V\right) \psi=0
$$

Tal como no caso da teoria de Dirac, a matriz $V$ pode ser escrita em termos de estruturas que exibem um comportamento bem definido segundo as transformações de Lorentz. Por causa da maior dimensão das matrizes $\beta^{\mu}$, comparada com as matrizes de Dirac, se espera um número maior de estruturas de Lorentz possíveis. De fato, para o setor escalar, as interações são constituídas de dois escalares, dois vetores e dois tensores, ao passo que para o setor vetorial, $V$ se apresenta como dois escalares, dois vetores, um pseudoescalar, dois pseudovetores e oito termos tensoriais.

Explicitamente, os covariantes bilineares do setor escalar têm a forma

- $\bar{\psi} \psi, \bar{\psi} \beta_{\mu} \beta^{\mu} \psi$ : que correspondem às interações do tipo escalar;

- $\bar{\psi} \beta^{\mu} \psi, i \bar{\psi}\left[P, \beta^{\mu}\right] \psi$ : que correspondem às interações do tipo vetorial;

- $\bar{\psi}\left\{\beta^{\mu}, \beta^{\nu}\right\} \psi$ : que corresponde à interação do tipo tensorial simétrica;

- $i \bar{\psi}\left[\beta^{\mu}, \beta^{\nu}\right] \psi:$ que corresponde à interação do tipo tensorial antissimétrica.

E os covariantes bilineares do setor vetorial têm a forma

- $\bar{\psi} \psi, \bar{\psi} \beta_{\mu} \beta^{\mu} \psi$ : que correspondem às interações do tipo escalar;

- $\bar{\psi} \epsilon_{\kappa \lambda \mu \nu} \beta^{\kappa} \beta^{\lambda} \beta^{\mu} \beta^{\nu} \psi$ : que corresponde à interação do tipo pseudoescalar;

- $\bar{\psi} \beta^{\mu} \psi, i \bar{\psi}\left[P, \beta^{\mu}\right] \psi$ : que correspondem às interações do tipo vetorial;

- $\bar{\psi} \epsilon_{\kappa \lambda \mu \nu} \beta^{\lambda} \beta^{\mu} \beta^{\nu} \psi, \quad i \epsilon_{\kappa \lambda \mu \nu} \bar{\psi}\left[\beta_{\sigma} \beta^{\sigma}, \beta^{\lambda} \beta^{\mu} \beta^{\nu}\right] \psi$ : que correspondem às interações do tipo pseudovetorial; 
- $\bar{\psi}\left[\left\{\beta^{\mu}, \beta^{\nu}\right\}-\frac{1}{2} g^{\mu \nu} \beta_{\sigma} \beta^{\sigma}\right] \psi, \bar{\psi}\left[\left\{\beta_{\sigma} \beta^{\sigma},\left\{\beta^{\mu}, \beta^{\nu}\right\}\right\}-g^{\mu \nu}\left(\beta_{\sigma} \beta^{\sigma}\right)^{2}\right] \psi$ : que correspondem às interações do tipo tensorial simétrica;

- $\bar{\psi}\left[\left\{\beta^{\mu}, \epsilon_{\lambda \sigma \nu}^{\kappa} \beta^{\lambda} \beta^{\sigma} \beta^{\nu}\right\}+\left\{\beta^{\kappa}, \epsilon_{\lambda \sigma \nu}^{\mu} \beta^{\lambda} \beta^{\sigma} \beta^{\nu}\right\}\right] \psi$ : que corresponde à interação do tipo pseudotensorial simétrica;

- $i \bar{\psi}\left[\beta^{\mu}, \beta^{\nu}\right] \psi, i \bar{\psi}\left\{\beta_{\sigma} \beta^{\sigma},\left[\beta^{\mu}, \beta^{\nu}\right]\right\} \psi$ : que correspondem às interações do tipo tensorial antissimétrica;

- $\bar{\psi} T^{\mu \nu \rho} \psi, i \bar{\psi}\left\{\beta_{\sigma} \beta^{\sigma}, T^{\mu \nu \rho}\right\} \psi, \bar{\psi} Y^{\kappa \lambda \mu \nu} \psi$ : que correspondem às interações do tipo tensorial,

em que

$$
T^{\mu \nu \rho} \equiv i\left\{\beta^{\mu},\left[\beta^{\nu}, \beta^{\rho}\right]\right\}+\frac{2}{3} \epsilon^{\mu \nu \rho \kappa} \epsilon_{\kappa \lambda \sigma \pi} \beta^{\lambda} \beta^{\sigma} \beta^{\pi}-\frac{i}{3} \delta^{\mu \nu}\left[\beta_{\sigma} \beta^{\sigma}, \beta^{\rho}\right]+\frac{i}{3} \delta^{\mu \rho}\left[\beta_{\sigma} \beta^{\sigma}, \beta^{\nu}\right]
$$

e

$$
\begin{aligned}
Y^{\kappa \lambda \mu \nu} \equiv & \left\{\left[\beta^{\kappa}, \beta^{\mu}\right],\left[\beta^{\lambda}, \beta^{\nu}\right]\right\}-\left\{\left[\beta^{\kappa}, \beta^{\nu}\right],\left[\beta^{\lambda}, \beta^{\mu}\right]\right\}-\frac{1}{2} \delta^{\kappa \mu}\left\{\left[\beta^{\rho}, \beta^{\nu}\right],\left[\beta_{\rho}, \beta^{\lambda}\right]\right\} \\
& -\frac{1}{2} \delta^{\lambda \nu}\left\{\left[\beta^{\rho}, \beta^{\mu}\right],\left[\beta_{\rho}, \beta^{\kappa}\right]\right\}+\frac{1}{2} \delta^{\kappa \nu}\left\{\left[\beta^{\rho}, \beta^{\mu}\right],\left[\beta_{\rho}, \beta^{\lambda}\right]\right\}+\frac{1}{2} \delta^{\lambda \mu}\left\{\left[\beta^{\rho}, \beta^{\nu}\right],\left[\beta_{\rho}, \beta^{\kappa}\right]\right\} \\
& +\frac{1}{6}\left(\delta^{\kappa \mu} \delta^{\lambda \nu}-\delta^{\kappa \nu} \delta^{\lambda \mu}\right)\left\{\left[\beta^{\rho}, \beta^{\alpha}\right],\left[\beta_{\rho}, \beta_{\alpha}\right]\right\}
\end{aligned}
$$

Em verdade, os termos tensoriais são evitados em aplicações porque fornecem efeitos não-causais [21,22].

Uma vez que as interações possíveis para os formalismos de Klein-Gordon-Fock e Proca são do tipo escalar e vetorial, em princípio, é possível que a teoria de DKP possa descrever fenômenos físicos incapazes de serem descritos nestes formalismos [23 25]. De fato, esta seria uma prova cabal da não-equivalência entre os formalismos.

Por fim, na presença de interações, a equação de DKP conjugada se torna

$$
i \partial_{\mu} \psi^{\dagger} \eta^{0} \beta^{\mu}+m \psi^{\dagger} \eta^{0}+\psi^{\dagger} \eta^{0} \eta^{0} V^{\dagger} \eta^{0}=0
$$

e, uma vez que $\psi^{\dagger} \eta^{0}=\bar{\psi}$

$$
i \partial_{\mu} \bar{\psi} \beta^{\mu}+m \bar{\psi}+\bar{\psi} \eta^{0} V^{\dagger} \eta^{0}=0
$$

A equação da continuidade na teoria de DKP com interações gerais é obtida de maneira análoga ao caso livre 26, 27, multiplicando (169) por $\bar{\psi}$ à esquerda, 170 por $\psi$ à direita e somando ambas as equações resultantes, obtendo

$$
i \partial_{\mu} \bar{\psi} \beta^{\mu} \psi+\bar{\psi}\left(\eta^{0} V^{\dagger} \eta^{0}-V\right) \psi=0
$$

$\operatorname{mas} J^{\mu} \equiv \bar{\psi} \beta^{\mu} \psi ; \log \mathrm{o}$

$$
\partial_{\mu} J^{\mu}+i \bar{\psi}\left(V-\eta^{0} V^{\dagger} \eta^{0}\right) \psi=0
$$

e a quadricorrente será conservada apenas quando $V$ for hermitiano com respeito a $\eta^{0}$. Isso se deve ao fato de que se trata de uma forma bilinear. Uma forma bilinear é uma generalização do conceito de produto interno, e é bem conhecida na teoria de Dirac por tratar-se de uma estrutura com comportamento bem definido sob transformações de Lorentz, e então chamados covariantes bilineares.

\section{Comentários finais}

Este estudo sobre a teoria de Duffin-KemmerPetiau abordou principalmente as motivações (ou inspirações) e os principais personagens que possibilitaram o advento de uma teoria relativística em primeira ordem para partículas de spin 0 e spin 1. No entanto, cabe ainda ressaltar que esta busca foi também tomada de forma independente por Sakata e Taketani, mas a prisão de Taketani por razões políticas manteve o trabalho latente. Tamm, Feshbach e Villars, Lubanksi, Bhabha, Madvarao e Harish-Chandra são também personagens que permearam o desenvolvimento de teorias relativísticas para a descrição de partículas elementares de spin mais altos e generalizações da teoria relativística em primeira ordem ${ }^{6}$ [1], e marcaram a década de 40.

Embora a teoria de DKP não seja popular devido ao crescente número de estudos evidenciando processos eletrodinâmicos nos quais ela era equivalente à teoria de Klein-Gordon-Fock, sua abordagem em primeira ordem pode ainda ser preferível pela ausência

${ }^{6}$ Por curiosidade: a decisão de usar a palavra méson (para partículas de Yukawa) foi feita na casa de Egon Bretscher por Maurice Price, Homi Bhabha e Kemmer (carta de N. Kemmer a M. M. Nieto datada de 27 de abril de 1976). 
de termos derivativos na interação eletromagnética. Mais ainda, o estudo da eletrodinâmica escalar via teoria de DKP apresenta uma considerável similaridade estrutural com a eletrodinâmica quântica (QED), o que torna seu estudo congênere [28. Na física nuclear, a teoria ainda têm se mostrado profícua no que concerne à fenomenologia, uma vez que a vasta gama de interações presentes na teoria de DKP são impossíveis de serem expressas pelas teorias de Klein-Gordon-Fock ou Proca [29]. A teoria de DKP também experimentou novo fôlego no contexto de aplicações em cromodinâmica quântica em curtas e longas distâncias por Gribov [30], no espalhamento de núcleos $K^{+}$[31], dinâmica hamiltoniana covariante [32, espaço-tempo curvo [33], invariância de Galileu em cinco dimensões [34], no contexto da invariância de gauge clássica [35, no método causal de Epstein-Glaser [36] e, sistematicamente, no estudo de espalhamento e confinamento de partículas [37.

\section{Apêndice - Uma representação para as matrizes $\beta^{\mu}$}

No presente estudo é adotada uma representação para as matrizes $\beta^{\mu}$ nas quais elas são reais.

Uma representação para as matrizes $\beta^{\mu}$ do setor escalar pode ser escrita como

$$
\begin{aligned}
\beta^{0}=\left(\begin{array}{ccccc}
0 & 1 & 0 & 0 & 0 \\
1 & 0 & 0 & 0 & 0 \\
0 & 0 & 0 & 0 & 0 \\
0 & 0 & 0 & 0 & 0 \\
0 & 0 & 0 & 0 & 0
\end{array}\right), & \beta^{1}=\left(\begin{array}{cccccc}
0 & 0 & 1 & 0 & 0 \\
0 & 0 & 0 & 0 & 0 \\
0 & 0 & 0 & 0 & 0 \\
0 & 0 & 0 & 0 & 0 \\
-1 & 0 & 0 & 0 & 0
\end{array}\right), \\
\beta^{2}=\left(\begin{array}{ccccc}
0 & 0 & 0 & 1 & 0 \\
0 & 0 & 0 & 0 & 0 \\
0 & 0 & 0 & 0 & 0 \\
-1 & 0 & 0 & 0 & 0 \\
0 & 0 & 0 & 0 & 0
\end{array}\right), & \beta^{3}=\left(\begin{array}{ccccc}
0 & 0 & 0 & 0 & 1 \\
0 & 0 & 0 & 0 & 0 \\
0 & 0 & 0 & 0 & 0 \\
0 & 0 & 0 & 0 & 0 \\
-1 & 0 & 0 & 0 & 0
\end{array}\right)
\end{aligned}
$$

Uma representação para as matrizes $\beta^{\mu}$ do setor vetorial pode ser escrita como

$$
\begin{aligned}
& \beta^{0}=\left(\begin{array}{cccccccccc}
0 & 0 & 0 & 0 & 0 & 0 & 0 & 0 & 0 & 0 \\
0 & 0 & 0 & 0 & 0 & 0 & 0 & 1 & 0 & 0 \\
0 & 0 & 0 & 0 & 0 & 0 & 0 & 0 & 1 & 0 \\
0 & 0 & 0 & 0 & 0 & 0 & 0 & 0 & 0 & 1 \\
0 & 0 & 0 & 0 & 0 & 0 & 0 & 0 & 0 & 0 \\
0 & 0 & 0 & 0 & 0 & 0 & 0 & 0 & 0 & 0 \\
0 & 0 & 0 & 0 & 0 & 0 & 0 & 0 & 0 & 0 \\
0 & 1 & 0 & 0 & 0 & 0 & 0 & 0 & 0 & 0 \\
0 & 0 & 1 & 0 & 0 & 0 & 0 & 0 & 0 & 0 \\
0 & 0 & 0 & 1 & 0 & 0 & 0 & 0 & 0 & 0
\end{array}\right), \quad \beta^{1}=\left(\begin{array}{cccccccccc}
0 & 0 & 0 & 0 & 0 & 0 & 0 & -1 & 0 & 0 \\
0 & 0 & 0 & 0 & 0 & 0 & 0 & 0 & 0 & 0 \\
0 & 0 & 0 & 0 & 0 & 0 & -1 & 0 & 0 & 0 \\
0 & 0 & 0 & 0 & 0 & 1 & 0 & 0 & 0 & 0 \\
0 & 0 & 0 & -1 & 0 & 0 & 0 & 0 & 0 & 0 \\
0 & 0 & 1 & 0 & 0 & 0 & 0 & 0 & 0 & 0 \\
0 & 0 & 0 & 0 & 0 & 0 & 0 & 0 & 0 & 0 \\
1 & 0 & 0 & 0 & 0 & 0 & 0 & 0 & 0 & 0 \\
0 & 0 & 0 & 0 & 0 & 0 & 0 & 0 & 0 & 0 \\
0 & 0 & 0 & 0 & 0 & 0 & 0 & 0 & 0 & 0
\end{array}\right), \\
& \beta^{2}=\left(\begin{array}{cccccccccc}
0 & 0 & 0 & 0 & 0 & 0 & 0 & 0 & -1 & 0 \\
0 & 0 & 0 & 0 & 0 & 0 & 1 & 0 & 0 & 0 \\
0 & 0 & 0 & 0 & 0 & 0 & 0 & 0 & 0 & 0 \\
0 & 0 & 0 & 0 & -1 & 0 & 0 & 0 & 0 & 0 \\
0 & 0 & 0 & 1 & 0 & 0 & 0 & 0 & 0 & 0 \\
0 & 0 & 0 & 0 & 0 & 0 & 0 & 0 & 0 & 0 \\
0 & -1 & 0 & 0 & 0 & 0 & 0 & 0 & 0 & 0 \\
0 & 0 & 0 & 0 & 0 & 0 & 0 & 0 & 0 & 0 \\
1 & 0 & 0 & 0 & 0 & 0 & 0 & 0 & 0 & 0 \\
0 & 0 & 0 & 0 & 0 & 0 & 0 & 0 & 0 & 0
\end{array}\right), \quad \beta^{3}=\left(\begin{array}{cccccccccc}
0 & 0 & 0 & 0 & 0 & 0 & 0 & 0 & 0 & -1 \\
0 & 0 & 0 & 0 & 0 & -1 & 0 & 0 & 0 & 0 \\
0 & 0 & 0 & 0 & 1 & 0 & 0 & 0 & 0 & 0 \\
0 & 0 & 0 & 0 & 0 & 0 & 0 & 0 & 0 & 0 \\
0 & 0 & -1 & 0 & 0 & 0 & 0 & 0 & 0 & 0 \\
0 & 1 & 0 & 0 & 0 & 0 & 0 & 0 & 0 & 0 \\
0 & 0 & 0 & 0 & 0 & 0 & 0 & 0 & 0 & 0 \\
0 & 0 & 0 & 0 & 0 & 0 & 0 & 0 & 0 & 0 \\
0 & 0 & 0 & 0 & 0 & 0 & 0 & 0 & 0 & 0 \\
1 & 0 & 0 & 0 & 0 & 0 & 0 & 0 & 0 & 0
\end{array}\right),
\end{aligned}
$$




\section{Agradecimentos}

T.R.C. agradece à CAPES pelo suporte financeiro e B.M.P. agradece ao $\mathrm{CNPq}$ pelo suporte financeiro parcial.

\section{Referências}

[1] J.J. Sakurai, Advanced Quantum Mechanics (Addison-Wesley, California, 1967).

[2] G.W. Series, Spectrum of atomic hydrogen (Oxford University Press, London, 1957).

[3] N. Bohr, Philosophical Magazine Series 6, 26, 1 (1913).

[4] E. Schrödinger, Physical Review 6, 28 (1926).

[5] P. Dirac, Proceedings of the Royal Society of London 117, 610 (1928).

[6] A. Piza, Mecânica Quântica (Edusp, São Paulo, 2002).

[7] W. Pauli and W.F. Weisskopf, Helvetica Physica Acta 1, 709 (1934).

[8] L. de Broglie, Comptes Rendus de l'Académie des Sciences 199, 445 (1934).

[9] J. Géhéniau, Académie Royale de Belgique 18 (1938).

[10] G. Petiau, Académie Royale de Belgique 16 (1936).

[11] R.A. Krajcik and M.M. Nieto, American Journal of Physics 45, 818 (1977).

[12] R.J. Duffin, Physical Review 54, 1114 (1938).

[13] C.A.M. de Melo, Geometria Invariante de Escala. Tese de doutorado do Instituto de Física Teórica, Unesp, disponível em http://repositorio.unesp. $\mathrm{br} / \mathrm{handle} / 11449 / 91853$.

[14] R.I.A.C. Astiazarán, The Kemmer Equation for Pion-Nucleus Scattering. Thesis, McGrill University, Montréal (1991).

[15] H. Umezawa, Quantum Field Theory (NorthHolland, Amsterdan, 1956).

[16] I. Fugiwara, Progress of Theoretical Physics 10, 589 (1953).

[17] D.C. Peaslee, Progress of Theoretical Physics 6, 639 (1951).

[18] M. Nowakowski, Physics Letters A 244, 329 (1998).

[19] J.T. Lunardi, B.M. Pimentel, R.G. Teixeira and J.S. Valverde, Physics Letters A 268, 165 (2000).

[20] Harish-Chandra, Proceedings of the Royal Society A 186, 502 (1946).

[21] R.F. Guertin and T.L. Wilson, Physical Review D 15, 1518 (1977).

[22] B. Vijayalakshmi, M. Seetharaman and P.M. Mathews, Journal of Physics A 12, 665 (1979).

[23] B.C. Clark, S. Hama, G.R. Kälbermann, R.L. Mercer and L. Ray, Physical Review Letters 55, 592 (1985).

[24] S. Ait-Tahar, J.S. Al-Khalili and Y. Nedjadi, Nuclear Physics A 589, 307 (1995).
[25] B.C. Clark, R.J. Furnstahl, L.J. Kurth-Kerr and S. Hama, Physics Letters B 427, 321 (1993).

[26] T.R. Cardoso, L.B. Castro and A.S. de Castro, Physics Letters A 372, 5964 (2008).

[27] T.R. Cardoso, Sobre Interações Escalares e Vetoriais na Teoria de Duffin-Kemmer-Petiau. Dissertação de Mestrado, Faculdade de Engenharia de Guaratinguetá, Unesp, disponível em http://repositorio. unesp.br/handle/11449/91805.

[28] R. Bufalo, T.R. Cardoso, A.A. Nogueira and B.M. Pimentel, arXiv:1510.04877.

[29] E. Fischbach and M.M. Nieto, Physical Review Letters 29, 1046 (1972); N.G. Deshpande and P.C. McNamee, Physical Review D 5, 1012 (1972); A.O. Barut and Z.Z. Aydin, Physical Review D 6, 3340 (1972); E. Fischbach, M.M. Nieto and C.K. Scott, Physical Review D 7, 207 (1973); Z.Z. Aydin and A.O. Barut, Physical Review D 7, 3522 (1973).

[30] V. Gribov, The European Physical Journal C 10, 71 (1999); V. Gribov, The European Physical Journal C 10, 91 (1999); J. Nyiri, The Gribov Theory of Quark Confinement (World Scientific Publishing, Singapura, 2001).

[31] L.K. Kerr, B.C. Clark, S. Hama, L. Ray and G.W. Hoffmann, Progress of Theoretical Physics 103, 321 (2000).

[32] I.V. Kanatchikov, Reports on Mathematical Physics 46, 1 (2000).

[33] R. Casana, J.T. Lunardi, B.M. Pimentel and R.G. Teixeira, General Relativity and Gravitation 34, 491 (2002); R. Casana, J.T. Lunardi, B.M. Pimentel and R.G. Teixeira, International Journal of Modern Physics A 17, 4197 (2002); R. Casana, J.T. Lunardi, B.M. Pimentel and R.G. Teixeira, Classical and Quantum Gravity 22, 3083 (2005); R. Casana, V. Ya. Fainberg, J.T. Lunardi and B.M. Pimentel, Classical and Quantum Gravity 20, 2457 (2003); R. Casana, C.A.M. de Melo and B.M. Pimentel, Classical and Quantum Gravity 24, 723 (2007).

[34] M. de Montigny, F.C. Khanna, A.E. Santana, E.S. Santos and J.D.M. Vianna, Journal of Physics A 33, 273 (2000);

[35] J.T. Lunardi, B.M. Pimentel, R.G. Teixeira and J.S. Valverde, Physics Letters A 268, 165 (2000).

[36] J.T. Lunardi, L.A. Manzoni, B.M. Pimentel and J.S. Valverde, International Journal of Modern Physics A 17, 205 (2002).

[37] T.R. Cardoso, L.B. Castro and A.S. de Castro, Canadian Journal of Physics 87, 857 (2009); T.R. Cardoso, L.B. Castro and A.S. de Castro, Canadian Journal of Physics 87, 1185 (2009); T.R. Cardoso, L.B. Castro and A.S. de Castro, International Journal of Theoretical Physics 49, 10 (2010); T.R. Cardoso, L.B. Castro and A.S. de Castro, Nuclear Physics B, Proceedings Supplement 199, 207 (2010); T.R. Cardoso, L.B. Castro and A.S. de Castro, Journal of Physics A, Mathematical and Theoretical 43, 055306 (2010). 